\title{
The K giant star Arcturus: the hybrid nature of its infrared spectrum ${ }^{\star}$
}

\author{
T. Tsuji \\ Institute of Astronomy, School of Sciences, The University of Tokyo, Mitaka, Tokyo, 181-0015, Japan \\ e-mail: ttsuji@ioa.s.u-tokyo.ac.jp
}

Received 14 April 2009 / Accepted 28 May 2009

ABSTRACT

\begin{abstract}
Aims. We investigate the infrared spectrum of Arcturus to clarify the nature of the cool component of its atmosphere, referred to as the CO-mosphere, and its relationship to the warm molecular envelope or the MOLsphere in cooler M (super)giant stars.

Methods. We apply the standard methods of spectral analysis to the CO lines measured from the "Infrared Atlas of the Arcturus Spectrum" by Hinkle, Wallace, and Livingston.

Results. We found that the intermediate-strength lines (with $-4.75<\log W / v \lesssim-4.4$ : $W$ is the equivalent width and $v$ the wavenumber) of $\mathrm{CO}$ fundamentals as well as overtones cannot be interpreted with the line-by-line analysis based on the classical line formation theory, while the weaker lines can and provide some information on the photosphere (e.g. $\log A_{\mathrm{C}}=7.97 / \log A_{\mathrm{H}}=12.00, \xi_{\text {micro }}=$ $1.87 \mathrm{~km} \mathrm{~s}^{-1}$, and $\xi_{\text {macro }}=3.47 \mathrm{~km} \mathrm{~s}^{-1}$ ). The nature of CO lines shows an abrupt change at $\log W / v \approx-4.75$ and the lines stronger than this limit indicate large excess absorption that cannot be photospheric in origin. This difficulty also appears as an unpredictable upturn (at $\log W / v \approx-4.75$ ) in the flat part of the curves-of-growth of $\mathrm{CO}$ lines. We confirm the same phenomenon in dozens of $\mathrm{M}$ giant stars, whose infrared spectra are known to have hybrid origins in the photosphere and extra-molecular constituent referred to as the MOLsphere. Thus the curve-of-growth analysis provides a simple means by which to recognize the hybrid nature of the infrared spectra. We note that the curves-of-growth of red supergiants and Mira variables found in the literature show similar peculiar patterns. The intermediate-strength lines of $\mathrm{CO}$ in Arcturus show only minor expansion $\left(\$ 0.6 \mathrm{~km} \mathrm{~s}^{-1}\right)$ relative to the weak lines and only small line-asymmetry, but the strong lines of the CO fundamentals exhibit higher turbulent velocity than the other CO lines.

Conclusions. The large excess absorption of the $\mathrm{CO}$ fundamental lines in Arcturus appears to be the same phenomenon as that found in the $\mathrm{CO}$ overtone lines of cooler M giant stars. Thus, molecular condensation referred to as the MOLsphere in cool luminous stars may also exist in Arcturus. The MOLsphere, however, is not necessarily a separate "sphere" but may be an aggregation of molecular clouds formed within the outer atmosphere. The formation of molecular clouds (referred to as MOLsphere in our modeling) in the outer atmosphere appears to be a basic feature of all the red giant stars from early $\mathrm{K}$ to late $\mathrm{M}$ types (and red supergiants).
\end{abstract}

Key words. stars: atmospheres - stars: chromospheres - stars: individual: Acturus - stars: late-type - stars: mass-loss - line: formation

\section{Introduction}

The $\mathrm{K}$ giant star Arcturus ( $\alpha$ Bootis: K1.5IIIp) is a prototype of cool giant stars because of its brightness and has been studied extensively with a variety of methods. Spectroscopic analyses have been facilitated greatly by the availability of high resolution spectral atlases; not only in the optical region (e.g. Griffin 1968) but also in the infrared region (Hinkle et al. 1995). Also, the other Arcturus atlases are reviewed by Hinkle et al. (1995).

The optical spectrum has been assumed to originate in the photosphere and its detailed analyses based on photospheric models have provided useful information on chemical abundances and photospheric structure among others. The infrared spectrum, however, appeared not to be so simple: As soon as high resolution spectra in the $5 \mu \mathrm{m}$-region could be observed by the Fourier Transform Spectroscopy (FTS) at Kitt Peak National Observatory (KPNO), it was noticed by Heasley et al. (1978) that the lines of the $\mathrm{CO}$ fundamental lines could not be interpreted with the photospheric model alone nor with the homogeneous model of the hot chromosphere based on the analysis of the CaII K line (Ayres \& Linsky 1975). The conclusion of this pioneering work about the $\mathrm{CO}$ fundamental lines was the possible

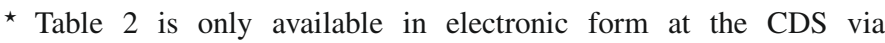
anonymous ftp to cdsarc.u-strasbg.fr (130.79.128.5) or via http://cdsweb.u-strasbg.fr/cgi-bin/qcat?]/A+A/504/543 presence of an inhomogeneous structure in the outer atmosphere of Arcturus (Heasley et al. 1978).

After a detailed analysis of the non-LTE effect on CO line formation (Ayres \& Wiedemann 1989; Wiedemann \& Ayres 1991), further analysis of CO fundamental lines by Wiedemann et al. (1994) revealed that no direct chromospheric indicator was present in the CO fundamental lines. The CO lines instead exhibited a steady decrease in temperature at the height where the chromospheric model shows a temperature increase. Thus these authors proposed a thermal bifurcation model for the outer atmosphere of Arcturus or a possible presence of a cool "COmosphere" in addition to the hot chromosphere. Additional evidence for a cool molecular constituent in Arcturus may be the detection of pure-rotation lines of $\mathrm{H}_{2} \mathrm{O}$ in the mid-infrared region by Ryde et al. (2002). Although these authors attributed the origin of the $\mathrm{H}_{2} \mathrm{O}$ lines possibly to an anomalous structure of the photosphere, this marvelous discovery may also be a manifestation of the cool constituent in the outer atmosphere of Arcturus. We should remember, however, that an exact relationship between the water vapor lines and neither the anomalous photospheric structure nor the cool molecular constituent is known yet.

The nature of the cool constituent, in either the photosphere or the outer atmosphere, has not yet been understood even in Arcturus, which is a star relatively well studied. On the other 
hand, more or less similar phenomena were known, even more clearly, in cooler giant and supergiant stars. For example, it was shown that the infrared spectra of $\mathrm{M}$ giant stars represent a hybrid of at least two components originating in the photosphere and a non-photospheric molecular constituent (Tsuji 2008). This result extended the idea of quasi-static molecular layers suggested previously by the analysis of the low excitation $\mathrm{CO}$ lines alone (Tsuji 1988). Also, $\mathrm{H}_{2} \mathrm{O}$ lines were found in the early M giant (Tsuji 2001) and supergiant stars (Woolf et al. 1964; Tsuji 2000a), which should not be cool enough to accommodate water in their photospheres. These results again implied the possible presence of an extra constituent referred to as a warm molecular envelope or a MOLsphere. Furthermore, direct evidence for molecular layers in red (super)giant stars was shown by the extensive observations with spatial interferometry (e.g. Quirrenbach et al. 1993; Perrin et al. 2004a, 2005, 2007).

We ask whether the inhomogeneous atmosphere or COmosphere considered for Arcturus and the warm molecular envelope or MOLsphere discussed for cooler M giant and supergiant stars are the same phenomenon or not. There are two possible ways to answer this question: First we can investigate the nature of the infrared spectrum of Arcturus in some detail and look for similarities with the spectra of cooler stars. Second we can investigate the $\mathrm{CO}$ fundamentals in $\mathrm{M}$ giant and supergiants to probe directly the region of chromospheric activities and look for similarities with the results for Arcturus. The second approach would certainly be attractive but would require formidable observational efforts if not impossible with present-day observational facilities. In contrast, the first approach can be readily adopted thanks to the high quality infrared spectral atlas of Arcturus by Hinkle et al. (1995), and we follow this approach in this paper.

\section{Input data}

We summarize the input data to be used in our analyses, namely the spectra we use (Sect. 2.1) and their measurements (Sect. 2.2), basic stellar parameters (Sect. 2.3), and models we apply (Sect. 2.4).

\subsection{Observed data}

We use the electronic version of the "Infrared Atlas of the Arcturus Spectrum" by Hinkle et al. (1995). We analyze the spectra ratioed to the telluric spectrum derived either from solar or lunar spectra. Thanks to this difficult processing, as discussed in detail by Hinkle et al. (1995), many lines especially of the CO fundamentals could be made available. The observations were repeated twice in general, one in winter and one in summer (at KPNO in the northern hemisphere), to measure as many lines as possible in the spectra for different geocentric velocity shifts.

We reproduce some detail of the spectra that we use in our Table 1 which was adopted from Table 3 of Hinkle et al. (1995) for easy reference. For the frequency bands 1867-1965 and $1965-2021 \mathrm{~cm}^{-1}$ in Table 1, only winter data are available. For the frequency bands 2021-2098 and 2119-2189 $\mathrm{cm}^{-1}$, both winter and summer data are available, and we treat them as independent data sets rather than using the sum of the two. For the frequency band 4000-6675 $\mathrm{cm}^{-1}$, we use only summer data, although a winter spectrum is also available, because a sufficient number of lines could be measured from the summer spectrum alone.
Table 1. Observed data from the Arcturus IR Atlas (Hinkle et al. 1995).

\begin{tabular}{ccccc}
\hline \hline Sp. ref. & $\begin{array}{c}\text { Freq. band } \\
\left(\mathrm{cm}^{-1}\right)\end{array}$ & $\begin{array}{c}\text { Res. } \\
\left(\mathrm{cm}^{-1}\right)\end{array}$ & Obs. date & $\begin{array}{c}\text { Shift } \\
\left(\mathrm{km} \mathrm{s}^{-1}\right)\end{array}$ \\
\hline 1 & $1867-1965$ & 0.02 & 1994 Jan. 9 & -30.52 \\
2 & $1965-2021$ & 0.02 & 1994 Jan. 21 & -30.52 \\
3 & $2021-2098$ & 0.02 & 1994 Jan. 7 & -31.66 \\
4 & $2119-2189$ & 0.02 & 1994 Jan. 7 & -31.39 \\
5 & $2021-2098$ & 0.02 & 1993 Jun. 7 & 14.63 \\
6 & $2119-2189$ & 0.02 & 1993 Jun. 7 & 14.90 \\
7 & $4000-6675$ & 0.04 & 1993 Jun. 5 & 14.33 \\
\hline
\end{tabular}

\subsection{Measurements}

The continuum level of the ratioed spectra was adjusted to unity by the authors of the atlas, but this may not imply that this level of unity can be used as the continuum to which we refer in the measurements of the line intensities. Many peaks are found above the level of unity, especially in the $M$ band region where disturbance by the atmospheric lines is most serious. We then detect the highest peak in every $2 \mathrm{~cm}^{-1}$ interval throughout the region of interest. Since we use the ratioed spectrum, it is possible that the detected apparent peaks are disturbed by atmospheric lines, and we exclude such peaks. Thus, we plot the peaks that are not seriously disturbed by the atmospheric absorption against wavenumber. We fit a smooth curve passing these peaks but not necessarily connecting all the peaks, since some peaks are apparently below the neighboring peaks. It is uncertain whether the continuum adopted in this way is a true continuum, but we have no other reference by which to measure equivalent widths.

With the fiducial "continuum" defined this way, we measure the line widths (FWHMs), depths, and equivalent widths (EWs) and the results are given in Table 2 (in electronic form at the CDS). We note that the instrumental effect on the line widths and depths has not yet been corrected in Table 2. We measure the summer and winter spectra for the wavelength range of 20212098 and 2119-2189 $\mathrm{cm}^{-1}$ (see Table 1) separately and the resulting EWs measured in both spectra are compared in Fig. 1, which may provide some idea of the accuracy of our EW measurement.

\subsection{Basic stellar parameters}

In Table 3, we summarize the basic stellar parameters of Arcturus used as input data in our modeling and analyses. We mainly follow a previous survey by Hinkle et al. (1995) summarized in their Table 2, and update some data only if more recent results are available. For example, the effective temperature of Arcturus appears to converge to $\approx 4300 \mathrm{~K}$ according to the recent result of angular diameter measurement at $H$ band where the opacity reaches its minimum (Lacour et al. 2008), and by the analysis of the spectral energy distribution (Griffin \& LynasGray 1999).

\subsection{Models of the photosphere and chromosphere}

We apply our model photosphere code, used mainly for cooler red (super)giant stars (e.g. Tsuji 1978), to Arcturus with the basic parameters discussed in Sect. 2.3. We assume the chemical abundances for 34 elements determined by Peterson et al. (1993). The resulting radiative equilibrium (RE) model starting integration from $\log \tau_{0}=-6.0\left(\tau_{0}\right.$ is the optical depth defined by the continuous opacity at $0.81 \mu \mathrm{m}$ ) is shown by the solid line in 


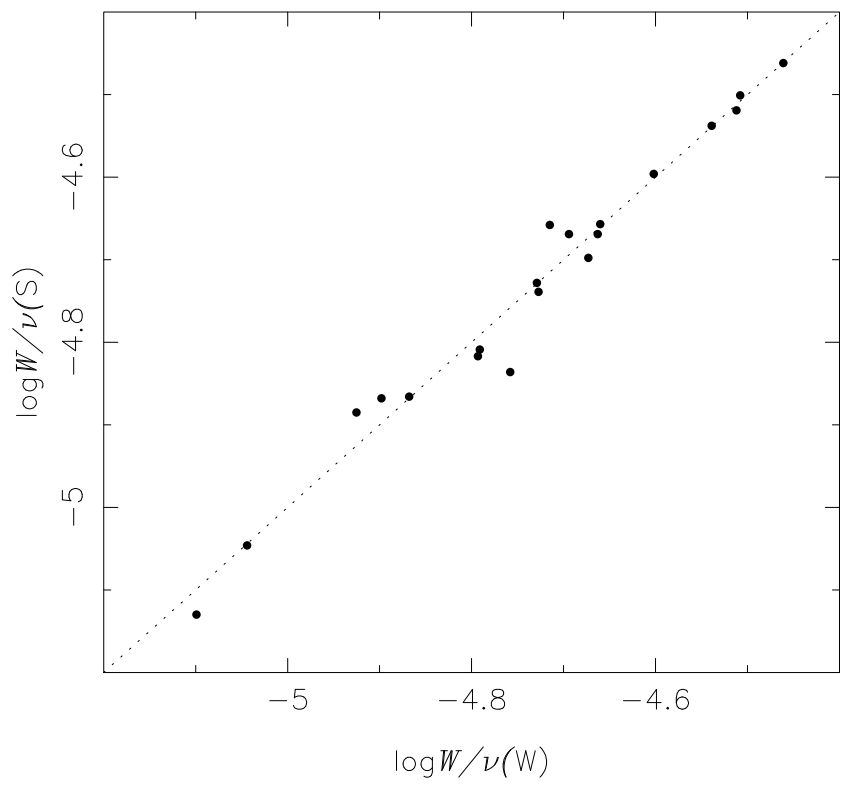

Fig. 1. The values of $\log W / v$ of the $\mathrm{CO}$ fundamental lines measured from the summer spectrum plotted against those from the winter spectrum.

Table 3. Basic stellar parameters of Arcturus.

\begin{tabular}{ll}
\hline \hline Parameter & Value \\
\hline angular diameter & $21.05 \pm 0.21\left(\right.$ mas $^{a}$ \\
effective temperature, $T_{\text {eff }}$ & $4300 \pm 30 \mathrm{~K}^{b}$ \\
surface gravity $(\log g)$ & $1.5 \pm 0.15^{b}$ \\
{$[\mathrm{Fe} / \mathrm{H}]$} & $-0.5 \pm 0.1^{b}$ \\
parallax & $88.85 \pm 0.74(\mathrm{mas})^{c}$ \\
radius, $R_{*}$ & $25.5 R_{\odot} \pm 1.5^{d}$ \\
mass, $M_{*}$ & $0.75 M_{\odot} \pm 0.2^{e}$ \\
micro turbulent velocity, $\xi_{\text {micro }}$ & $1.85 \mathrm{~km} \mathrm{~s}^{-1} f$ \\
radial velocity (heliocentric) & $-5.5 \mathrm{~km} \mathrm{~s}^{-1} g$ \\
\hline
\end{tabular}

${ }^{a}$ Lacour et al. (2008); ${ }^{b}$ Peterson et al. (1993); ${ }^{c}$ Hipparcos data (ESA 1997); ${ }^{d}$ based on the angular diameter and parallax; ${ }^{e}$ based on the radius and $\log g ;{ }^{f}$ based on our preliminary analysis (our final value is $\left.1.87 \mathrm{~km} \mathrm{~s}^{-1}\right) ;{ }^{g}$ Griffin \& Griffin (1973).

Fig. 2 (model a). Although our modeling code does not include atomic line-blanketing effect, our model agrees well with the model by Peterson et al. (1993) shown by the filled circles in Fig. 2; the largest difference is about $50 \mathrm{~K}$ at around $\tau_{0} \approx 10^{-3}$. This result implies that the thermal structure of the photosphere is largely determined by the blanketing effect of molecules rather than of atoms even in $K$ giants. We also compute a model assuming spherical symmetry (SS) rather than plane-parallel (PP). It is found that the differences between PP and SS models are rather minor for Arcturus photosphere, and a measure of the photospheric extension defined by $d=r\left(\tau_{0}=10^{-6}\right) / R_{*}$ (where $R_{*}$ is the stellar radius) is only 1.04. For this reason, we apply PP models to our spectral analysis in this paper.

We also compute an extended model photosphere (SS) by starting integration from $\log \tau_{0}=-9.0$ instead of -6.0 adopted in our modeling in general (e.g. model a). We assume a turbulent velocity of $6 \mathrm{~km} \mathrm{~s}^{-1}$ in the layers above $\log \tau_{0}=-6.0$ and the resulting RE model (model b in Fig. 2) is in hydrostatic equilibrium with turbulent pressure included (see e.g. Tsuji 2006). We note that the surface temperature is decreased below $2000 \mathrm{~K}$, although this has little effect on observed properties such as spectra, colors, and angular diameters, since the matter density is

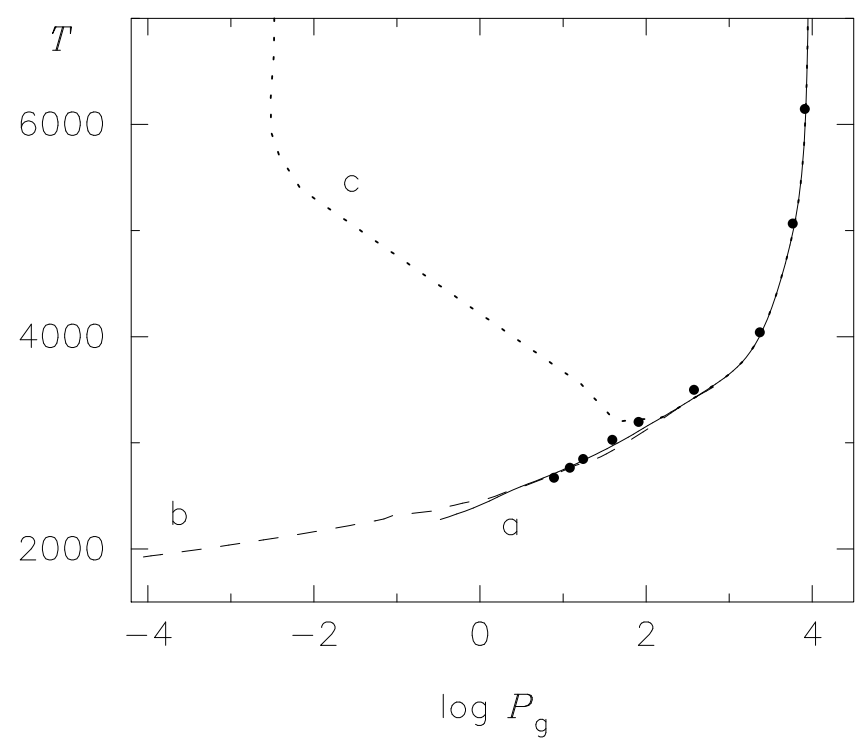

Fig. 2. Our RE model photosphere (plane parallel) of Arcturus shown by the solid line (model a) is compared with the RE model by Peterson et al. (1993) shown by the filled circles. Also, an extended RE model (spherically symmetric) of Arcturus starting integration from $\log \tau_{0}=$ -9.0 rather than from $\log \tau_{0}=-6.0$ (as in model a) is shown by the dashed line (model b). The chromospheric model by Ayres \& Linsky (1975) is shown by the dotted line (model c).

very low in the layers above $\log \tau_{0}=-6.0$. We discuss this model further in Sect. 6.2.

It is known that the photospheric model alone is insufficient for interpreting the $\mathrm{CO}$ fundamental lines, which are formed in the regime of the chromospheric activities. For this reason, we fit the empirical model of the chromosphere of Arcturus by Ayres \& Linsky (1975) to our photospheric PP model as shown in Fig. 2 (model c).

\section{Line-by-line analysis of CO}

We first apply a line-by-line (LL) analysis in which each observed EW is interpreted with the use of the classical microturbulent model of line formation. For this purpose, we apply our CO linelist based on spectroscopic (Guelachivili et al. 1983) and intensity (Chackerian \& Tipping 1983) data.

\subsection{The CO first and second overtone bands}

We apply a detailed line-by-line analysis to the CO lines of the first and second overtone bands. Given the model photosphere as in Sect. 2.4, a given equivalent width can be interpreted in terms of the abundance and microturbulent velocity. We start from the abundances by Peterson et al. (1993). In particular, the carbon abundance is $\log A_{\mathrm{C}}=8.06$ on the scale of $\log A_{\mathrm{H}}=12.00$ (we use this scale for chemical abundances throughout this paper). Then we assume a value of the microturbulent velocity and determine an abundance correction to the assumed value needed to explain the observed equivalent width. This can be determined from a relationship between a few trial values of the abundance correction and the resulting equivalent widths (i.e. from a segment of a curve-of-growth, or a mini curve-of-growth, for the single line we are to analyze). The resulting abundance corrections should be the same for all the lines used if a correct microturbulent velocity is assumed. We repeat this process until we 


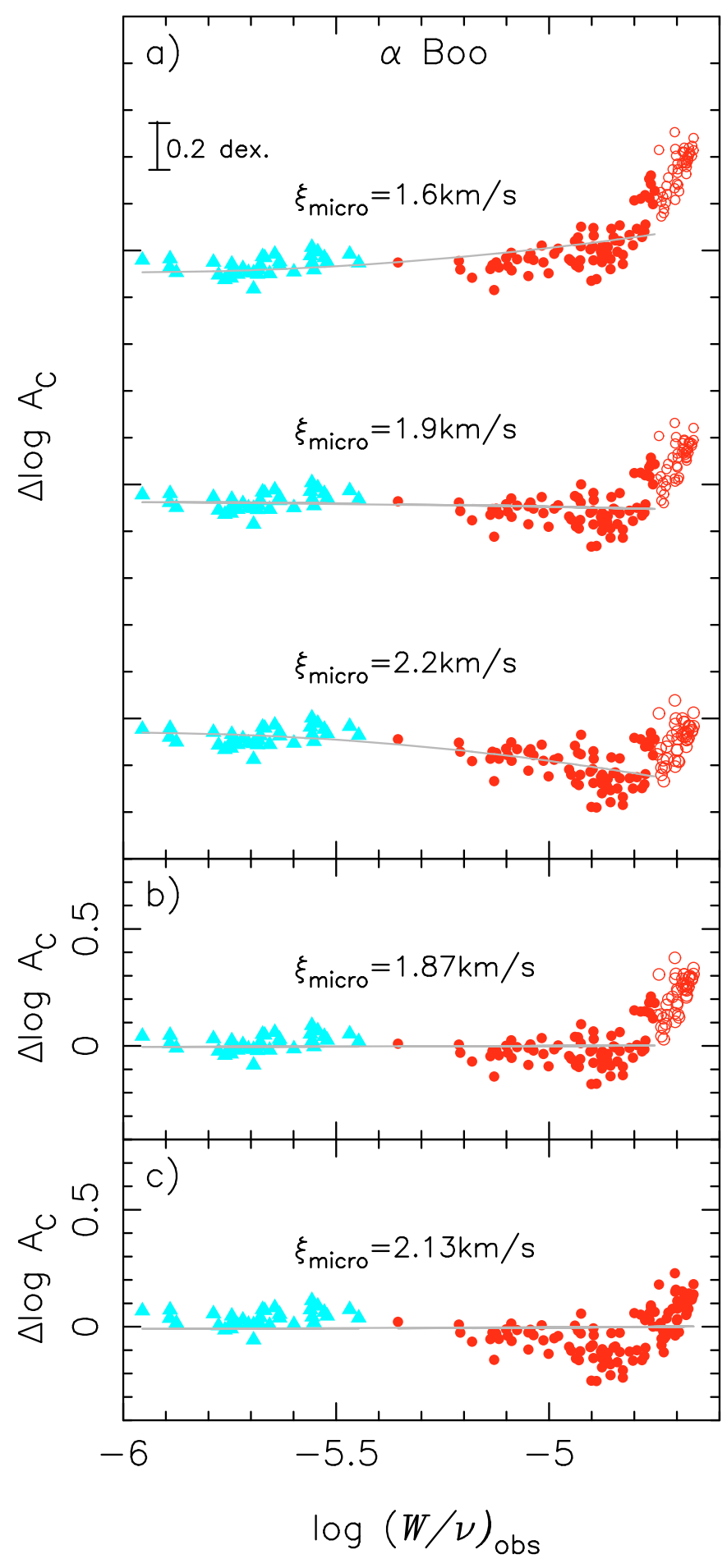

Fig. 3. a) Logarithmic abundance corrections for the lines of the $\mathrm{CO}$ overtone bands observed in $\alpha$ Boo plotted against $\log (W / v)_{\text {obs }}$ for assumed values of $\xi_{\text {micro }}=1.6,1.9$, and $2.2 \mathrm{~km} \mathrm{~s}^{-1}$. The CO first and second overtone lines are shown by the circles and triangles, respectively. Note that the intermediate-strength lines (shown by the open symbols) are not included in the analysis for the reason detailed in the text. (model photosphere: $T_{\text {eff }} / \log g / \xi_{\text {micro }}=4300 / 1.5 / 1.85$ ). b) Confirmation of the null $\operatorname{logarithmic}$ abundance corrections for $\log A_{\mathrm{C}}=7.97$ and $\xi_{\text {micro }}=1.87 \mathrm{~km} \mathrm{~s}^{-1}$, which are the solution of the LL analysis of the $\mathrm{CO}$ weak lines (shown by the filled symbols in a). c) Confirmation of the null $\operatorname{logarithmic}$ abundance corrections for $\log A_{\mathrm{C}}=7.94$ and $\xi_{\text {micro }}=2.13 \mathrm{~km} \mathrm{~s}^{-1}$, which are the formal solution of the LL analysis of all the $\mathrm{CO}$ overtone lines. Note that the groups of lines with different strengths show inconsistent abundance corrections even though the mean logarithmic abundance correction is null. find such a value of the microturbulent velocity (as for detail, see Tsuji 1986).

We show the resulting abundance corrections for the lines of the $\mathrm{CO}$ first (circles) and second (triangles) overtone bands obtained with the values of $\xi_{\text {micro }}=1.6,1.9$, and $2.2 \mathrm{~km} \mathrm{~s}^{-1}$ in Fig. 3a. For most lines, the results follow as expected from the classical line formation theory in that the abundance corrections are larger for smaller values of $\xi_{\text {micro }}$ for saturated lines. We note, however, that the lines of the $\mathrm{CO}$ first overtone bands stronger than $\log W / v \approx-4.75$ do not follow the expected behavior in that they never show abundance corrections consistent with those for the weaker lines for any assumed value of $\xi_{\text {micro }}$.

This is the same phenomenon that we have identified in many $\mathrm{M}$ giant stars, and we concluded that these lines, which we referred to as the intermediate-strength lines ${ }^{1}$, should be contaminated with contributions of non-photospheric origin (Tsuji 2008). We now confirm exactly the same phenomenon in the $\mathrm{K}$ giant star Arcturus. For this reason, we disregard the lines of $\log W / v>-4.75$ (i.e. the intermediate-strength lines) in our LL analysis, and obtain an abundance correction of $\Delta \log A=$ $-0.09 \pm 0.01$ and $\xi_{\text {micro }}=1.87 \pm 0.02 \mathrm{~km} \mathrm{~s}^{-1}$ from the lines with $\log W / v \lesssim-4.75$ (i.e. the weak lines). Since the $\mathrm{CO}$ abundance directly reflects the carbon abundance in oxygen-rich giants, we derive a revised value of $\log A_{C}=8.06-0.09=7.97$. We confirm in Fig. $3 \mathrm{~b}$ that this carbon abundance and $\xi_{\text {micro }}=$ $1.87 \mathrm{~km} \mathrm{~s}^{-1}$ provide a mean null logarithmic abundance correction.

If we include all the measured lines in our LL analysis, it is also possible to obtain formal solutions, which are $\Delta \log A=$ $-0.12 \pm 0.01$ and $\xi_{\text {micro }}=2.13 \pm 0.02 \mathrm{~km} \mathrm{~s}^{-1}$. The resulting carbon abundance of $\log A_{\mathrm{C}}=8.06-0.12=7.94$ and $\xi_{\text {micro }}=2.13 \mathrm{~km} \mathrm{~s}^{-1}$ provide a mean null logarithmic abundance correction as shown in Fig. 3c, but the different groups of lines, i.e., those of the $\mathrm{CO}$ second overtones, the weak lines of the $\mathrm{CO}$ first overtones, and the intermediate-strength lines of the $\mathrm{CO}$ first overtones, show large deviations from the null correction. This result confirms that it is not possible to carry out a consistent abundance analysis if we include the intermediate-strength lines in our LL analysis.

\subsection{The $C O$ fundamental bands}

We now include in our LL analysis the lines of the CO fundamentals shown by the squares in Fig. 4a. It appears that the stronger and weaker lines, divided at about $\log W / v \approx-4.75$, behave quite differently in the fundamentals more clearly than in the overtones. But the nature of the peculiar behavior of the stronger lines or the intermediate-strength lines is essentially the same as those lines of the overtones bands in Arcturus (see Fig. 3) as well as in many $\mathrm{M}$ giant stars (Tsuji 2008).

We again disregard the lines with $\log W / v>-4.75$ in the fundamentals as well as in the overtones in our LL analysis, and find $\Delta \log A=-0.11 \pm 0.01$ and $\xi_{\text {micro }}=2.01 \pm 0.03 \mathrm{~km} \mathrm{~s}^{-1}$. The revised carbon abundance of $\log A_{\mathrm{C}}=8.06-0.11=7.95$ together with $\xi_{\text {micro }}=2.01 \mathrm{~km} \mathrm{~s}^{-1}$ result in a null mean logarithmic abundance as confirmed in Fig. 4b. Thus, by including the fundamental lines with $\log W / v \lesssim-4.75$ in our LL analysis, $\Delta \log A$ and $\xi_{\text {micro }}$ change by $5 \%$ (from -0.09 to $-0.11 \mathrm{dex}$ ) and

1 We referred to the lines of $\log W / v \lessgtr-4.75$ as the weak lines (but not necessarily so weak as to be free from saturation effect), those with $-4.75<\log W / v \lesssim-4.4$ as the intermediate-strength lines, and those with $\log W / v>-4.4$ as the strong lines (Tsuji 2008), and we follow this classification in this paper. 
$7 \%$ (from 1.87 to $2.01 \mathrm{~km} \mathrm{~s}^{-1}$ ), respectively. For the origin of the differences caused by the inclusion of the fundamental bands, we already know that the effect of the hot chromosphere should be considered in the analysis of the $\mathrm{CO}$ fundamentals in Arcturus (Heasely et al. 1978; Wiedemann \& Ayres 1994). For this reason (and also for another reason to be discussed in Sect. 6.2), we propose that the results including the fundamental lines do not represent the photospheric values, and we adopt the results based on the weak lines of the overtone bands (Sect. 3.1) as the photospheric abundance (i.e. $\log A_{\mathrm{C}}=7.97 \pm 0.01$ ) and microturbulent velocity (i.e. $\xi_{\text {micro }}=1.87 \pm 0.02 \mathrm{~km} \mathrm{~s}^{-1}$ ) of Arcturus throughout this paper.

The behavior of the lines stronger than about $\log W / v \approx$ -4.75 cannot be explained at all by the effect of the hot chromosphere. It appears that the excess absorption, possibly of nonphotosphere origin, is more prominent in the stronger lines of the fundamentals than in the overtones. The detailed LL analysis outlined in this section, however, is not well optimized to the inhomogeneous models with cool and hot components, and we examine these cases with a different method in the next section.

\section{Curve-of-growth analysis}

It appears that the $\mathrm{CO}$ lines may not originate in the photosphere alone but may be disturbed not only by the effect of the hot chromosphere but also by other cool constituents as in M giant stars (Tsuji 2008). In these circumstances, we return to a simpler method based on the curve-of-growth by which all the observed equivalent widths are interpreted in terms of a single curve-of-growth for a set of representative parameters such as LEP (lower excitation potential), line position, and damping constant. In contrast, we essentially use different mini curves-ofgrowth for each individual lines with specified LEP, wavenumber, and damping constant in our detailed LL analysis ${ }^{2}$. Thus, the accuracy may be lower in the curve-of-growth (CG) analysis, but we hope to obtain a more intuitive view by adopting a simpler method.

\subsection{Arcturus}

In Fig. 5a, we plot the observed values of $\log W / v$ (from Table 2) of the CO first (filled circles) and second (filled triangles) overtone lines against

$\log (W / v)_{\mathrm{wk}}=\log g f+\log \Gamma_{v}(\chi)$,

where $\Gamma_{v}(\chi)$ is the line intensity integral evaluated by the weighting function method (for details, see the Appendix of Tsuji 1991). To this purpose, we apply our PP model photosphere (model a in Fig. 2) with the carbon abundance of $\log A_{\mathrm{C}}=7.97$ determined in Sect. 3.1. For comparison, we compute theoretical curves-of-growth for fictitious lines of LEP $=1 \mathrm{eV}$ and $\log g f=-7.00 \sim-2.00$ (by a step of 0.50 ) at $\lambda=2.30 \mu \mathrm{m}$ $\left(v=4348 \mathrm{~cm}^{-1}\right)$ for the CO first overtones (solid line), and at $\lambda=1.67 \mu \mathrm{m}\left(v=6000 \mathrm{~cm}^{-1}\right)$ for the CO second overtones (dashed line $)^{3}$. In the theoretical curve-of-growth, we assume

\footnotetext{
${ }^{2}$ We do not in fact consider the damping constants in detail but apply a simple analytical formula for collision half-width (Tsuji 1986), since collision damping plays a minor role in giant stars.

3 The theoretical curve-of-growth is defined as a relationship of $\log W / v$ against $\log (W / v)_{\mathrm{wk}}$ and converges to $\log W / v=\log (W / v)_{\mathrm{wk}}$ at the weak-line limit. The observed curve-of-growth is a plot of $\log (W / v)_{\mathrm{obs}}$ against $\log (W / v)_{\mathrm{wk}}$, and converges to $\log (W / v)_{\mathrm{obs}}=$ $\log (W / v)_{\mathrm{wk}}$ at the weak line limit only if the abundances assumed in evaluating $\log (W / v)_{\mathrm{wk}}$ are correct.
}

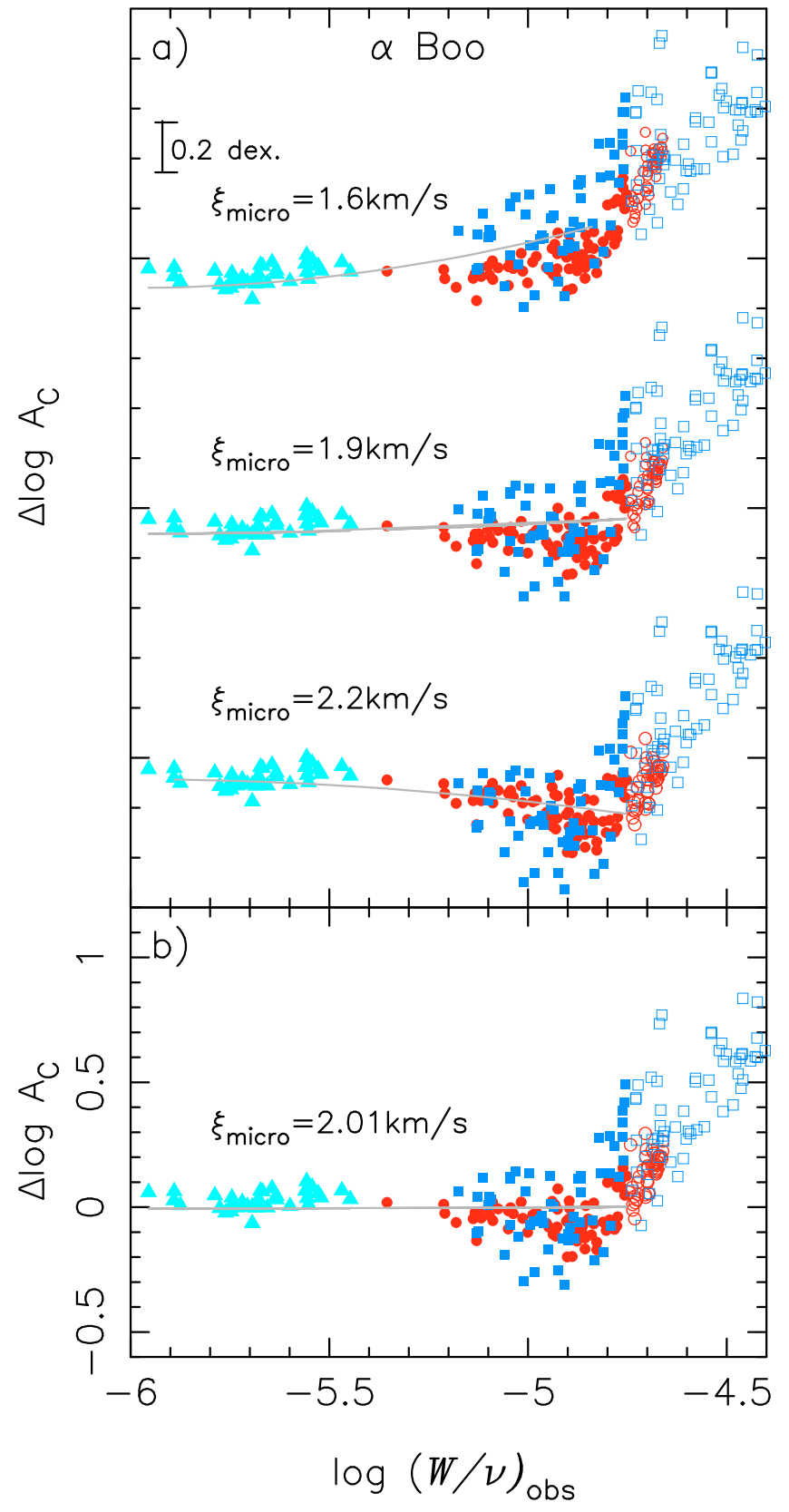

Fig. 4. a) The same as in Fig. 3a, but the lines of the CO fundamental bands shown by the squares are added to the LL analysis. Note that the intermediate-strength lines (shown by the open symbols) are again not included in the analysis. b) Confirmation of the null logarithmic abundance corrections for $\log A_{\mathrm{C}}=7.95$ and $\xi_{\text {micro }}=2.01 \mathrm{~km} \mathrm{~s}^{-1}$, which are the solution of the LL analysis of the weak lines of CO (shown by the filled symbols in a).

the microturbulent velocity of $\xi_{\text {micro }}=1.87 \mathrm{~km} \mathrm{~s}^{-1}$ determined in Sect. 3.1. The curves-of-growth for different lines should differ slightly depending on LEP and line position, and these differences are considered in our LL analysis. We recall, however, that these differences are ignored and one curve for representative parameters is used for all the lines in the classical CG method (coarse analysis).

Inspection of Fig. 5a reveals that the observed and theoretical curves-of-growth of the $\mathrm{CO}$ second overtones agree well in the weak line limit, and this fact confirms that the carbon abundance determined in Sect. 3.1 is supported by the most simple 

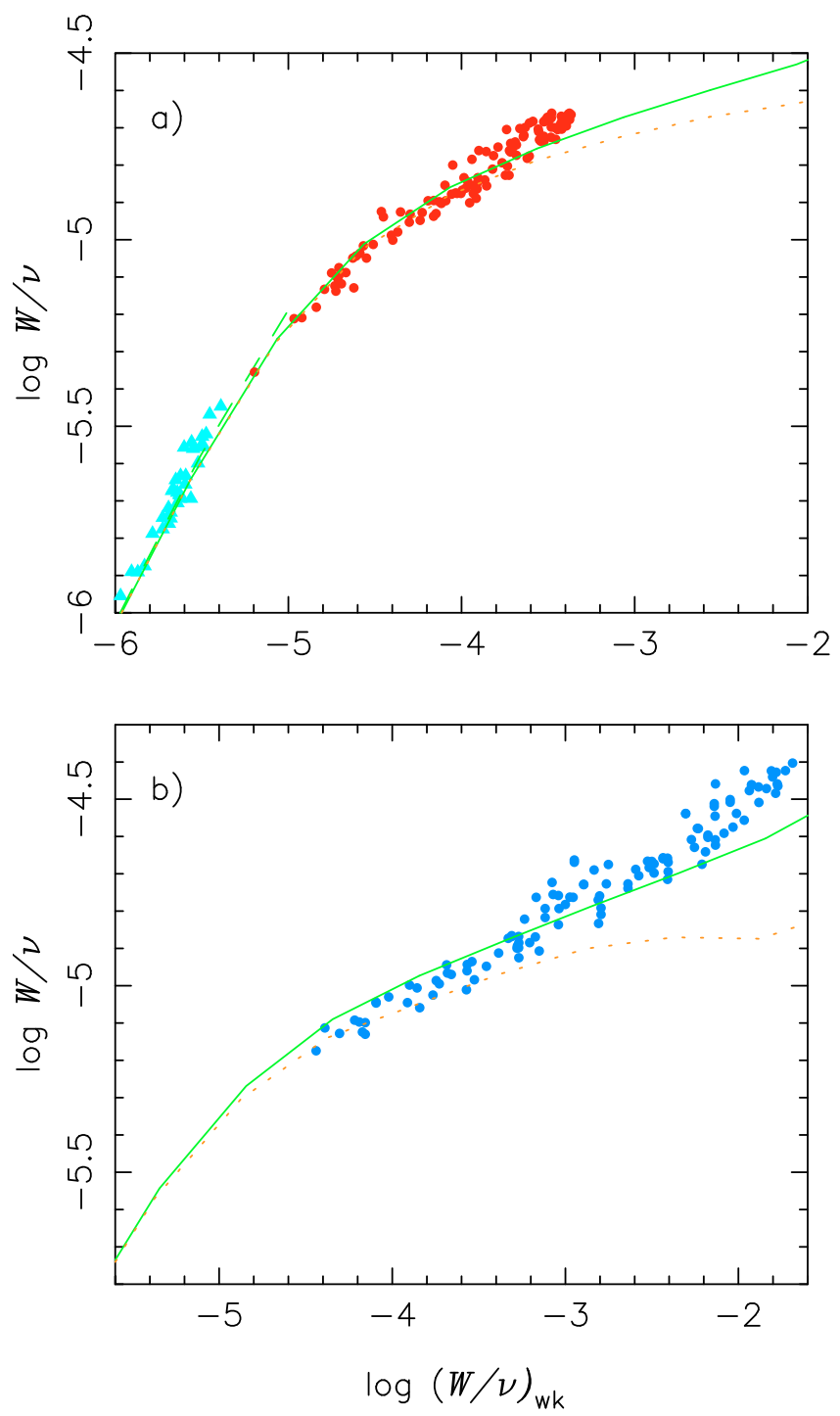

Fig. 5. a) Empirical curves-of-growth of the CO first (filled circles) and second (filled triangle) overtones observed in Arcturus are compared with the theoretical ones (solid and dashed lines for the first and second overtones, respectively) based on the PP model and the microturbulent velocity $\xi_{\text {micro }}=1.87 \mathrm{~km} \mathrm{~s}^{-1}$. The result for the homogeneous chromosphere shown in Fig. 2 is given by the dotted line. b) Empirical curveof-growth of the CO fundamentals (filled circles) is compared with the theoretical one (solid line) based on the PP model and the microturbulent velocity $\xi_{\text {micro }}=1.87 \mathrm{~km} \mathrm{~s}^{-1}$. The result for the homogeneous chromosphere is shown by the dotted line.

analysis of weak lines independently of the microturbulent model of line formation. The observed and theoretical curves-ofgrowth should also agree in the flat part of the curves-of-growth, if the microturbulent velocity assumed is correct. This expectation is fulfilled only partly in the region of $\log W / v \lessgtr-4.75$, namely at about the same region where our LL analysis could have been done consistently (Sect. 3.1). However, the empirical curve-of-growth deviates from the theoretical one in the region of $\log W / v>-4.75^{4}$. This result should be what can be expected from our LL analysis (Sect. 3.1), and the peculiar behavior of the

\footnotetext{
${ }^{4}$ In the classical CG analysis, the microturbulent velocity is determined by searching a best fit among theoretical curves-of-growth of different microturbulent velocities. Then it may be possible that the empirical curve-of-growth such as Fig. 5a may be fitted with a theoretical curve-of-growth of the microturbulent velocity somewhat larger
}

intermediate-strength lines is now confirmed by a simple curveof-growth analysis.

In Fig. 5b, we compare the empirical curve-of-growth for the $\mathrm{CO}$ fundamental lines (filled circles) with the theoretical one computed for fictitious lines of LEP $=1 \mathrm{eV}$ and $\log g f=$ $-7.00 \sim-2.00$ at $\lambda=5.0 \mu \mathrm{m}\left(v=2000 \mathrm{~cm}^{-1}\right)$ (solid line). We again use our PP model photosphere with $\log A_{\mathrm{C}}=7.97$ and $\xi_{\text {micro }}=1.87 \mathrm{~km} \mathrm{~s}^{-1}$ determined in Sect. 3.1. The observed curve-of-growth is slightly below the theoretical one for lines weaker than about $\log W / v \approx-4.85$ (about 0.1 dex lower than the critical value of -4.75 ) but shows opposite behavior for the stronger lines, consistent with the result of the LL analysis in Sect. 3.2.

So far, we have considered only the classical photosphere, but it has been known that the hot chromosphere should have some effects especially on the $\mathrm{CO}$ fundamentals formed in the upper photosphere (Heasely et al. 1978; Wiedemann \& Ayres 1994). We compute theoretical curves-of-growth for the empirical model of the hot homogeneous chromosphere (model c in Fig. 2) derived from the analysis of $\mathrm{Ca}$ II $\mathrm{H} \& \mathrm{~K}$ emission (Ayres \& Linsky 1975) discussed in Sect. 2.4, and the results are shown by the dotted lines in Fig. 5a,b. As expected, the lines are weakened by a temperature reversal in the chromospheric model (Fig. 2). The effect, however, is quite minor in the lines of the overtones, which are formed mostly below the temperature minimum (Fig. 5a). Departure from LTE in CO is also not important in the layers below the temperature minimum in Arcturus (Ayres \& Wiedemann 1989).

The effect of the hot chromosphere is increasingly prominent in the lines of the $\mathrm{CO}$ fundamentals (Fig. 5b). The observed curve-of-growth of the weaker lines appears between the theoretical curve-of-growth based on the classical RE model photosphere (solid line) and that based on the model of the homogeneous chromosphere (dotted line). This result may imply that the weaker lines of the CO fundamentals may be affected by the chromosphere, but only partly. This result is essentially the same as the previous result by Heaseley et al. (1978), in which the line profiles of the weaker lines of the $\mathrm{CO}$ fundamentals could not be understood by any single component model, neither homogeneous chromosphere nor a classical photosphere, but could be better described by the theoretical spectrum from a two-component model consisting of the hot chromosphere and cool photosphere.

The difficulty in interpreting the stronger lines of the $\mathrm{CO}$ fundamentals was discussed by Wiedemann \& Ayres (1992) and by Wiedemann et al. (1994), who showed that the strong lines of the $\mathrm{CO}$ fundamentals indicate very low surface temperature near $2400 \mathrm{~K}$ with no indication of the temperature increase expected by an empirical chromospheric model such as that of Ayres \& Linsky (1975). Based on a larger sample of CO lines, we arrive at a similar result in Fig. 5b: It is as if the possible weakening of the stronger lines by the chromospheric temperature increase is superseded by another effect producing very strong absorption. Thus, we agree with the conclusion of Wiedemann and his collaborators that the outer atmosphere of Arcturus is dominated by the cool gaseous component, which was referred to as the COmosphere by them. In view of this result that the effect of the

than $1.87 \mathrm{~km} \mathrm{~s}^{-1}$. With the result of the detailed line-by-line analysis in Sect. 3.1 at hand, we could avoid such an erroneous fit and we already know that the peculiar behavior of the intermediate-strength lines cannot be a problem of the value of the microturbulent velocity as noted in Sect. 3.1 (also see Tsuji 2008). This may be a reason why the LL analysis should be preferred to the much simpler classical CG analysis. 


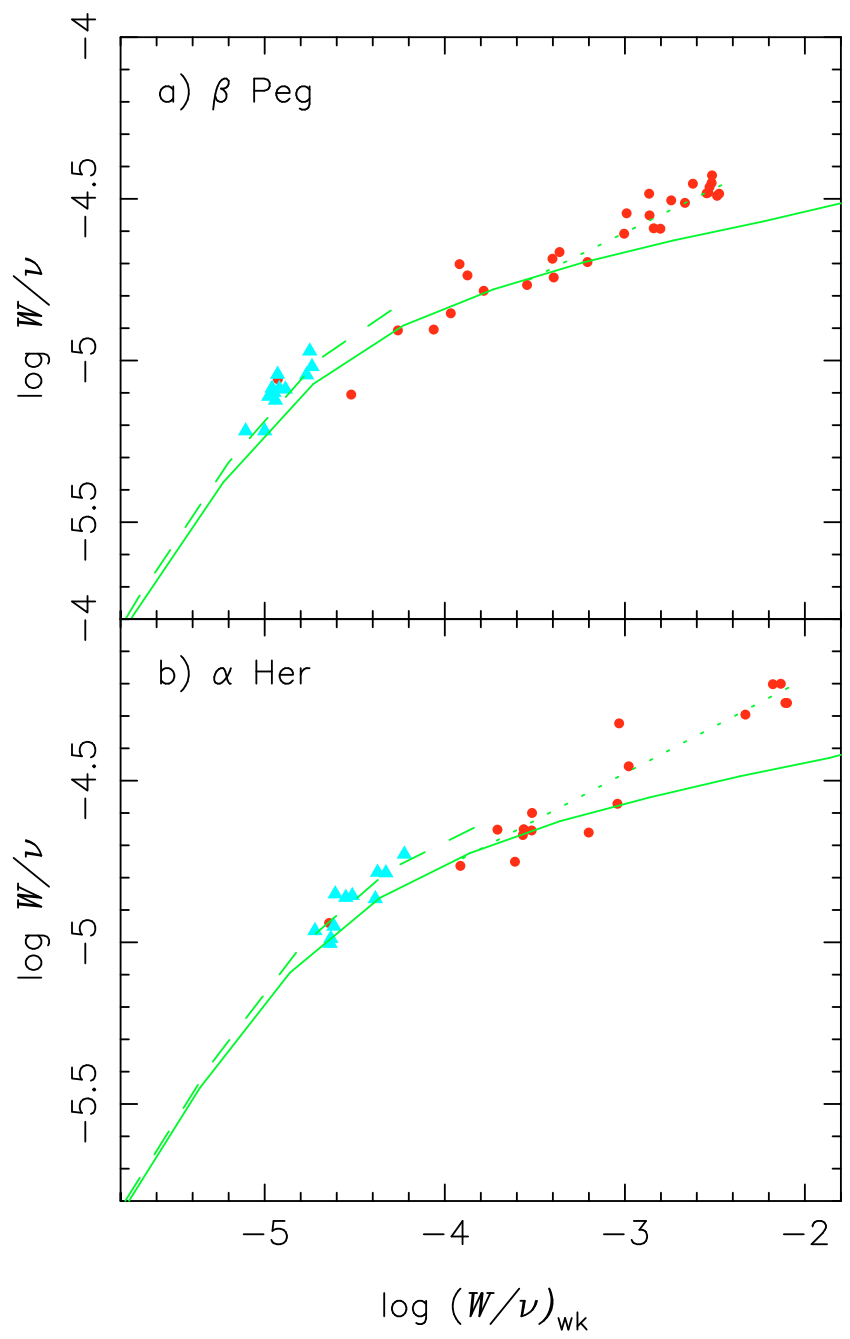

Fig. 6. Empirical curves-of-growth of the $\mathrm{CO}$ first (filled circles) and second (filled triangles) overtones are compared with the theoretical ones (solid and dashed lines are for the first and second overtones, respectively) based on the PP models and the microturbulent velocities given in Table 4. Note that the lines of the intermediate-strength show larger equivalent widths than those predicted by the theoretical curvesof-growth. a) $\beta$ Peg (M2.5II-III). b) $\alpha \operatorname{Her}$ (M5Ib-II).

chromosphere may not be significant, we also consider another interpretation of the weaker CO fundamental lines in Sect. 6.2, which we consider to be more likely.

So far we have assumed LTE in our analysis throughout, but we referred to the detailed analysis of the departure from LTE in CO line formation by Ayres \& Wiedemann (1989). They showed that the non-LTE effect in CO line formation is certainly non-negligible and even quite appreciable in the surface layers. However, their detailed numerical simulation of the CO lines of the fundamental bands showed that the non-LTE absorption cores of strong CO lines are slightly deeper than the LTE spectrum based on the RE model of Arcturus but are far too short to explain the observed strong CO lines. They also showed that non-LTE and LTE spectra for the chromospheric model (Ayres \& Linsky 1975) are almost identical ${ }^{5}$. Thus, our conclusion based

5 This unexpected result can be because $\mathrm{CO}$ lines are formed near the temperature minimum where departure from LTE is still minor and CO dissociates rapidly in the hot upper layers where non-LTE effect should be more significant. on an LTE analysis may be little affected by including a non-LTE effect, at least qualitatively.

\subsection{M-giant stars}

The unpredictable upturn in the curve-of-growth of the CO overtones (Fig. 5a) is rather subtle in the case of Arcturus, but the upturn may be more prominent in cooler M giant stars in which our LL analysis revealed large anomalies in lines stronger than $\log W / v \approx-4.75$ (Tsuji 2008). We now apply the CG analysis to $\mathrm{M}$ giants, since this should provide a simple means by which to investigate the nature of the excess absorption (or emission) in cool giant stars.

For this purpose, we apply the CG analysis to a few M giants by using PP models as in Arcturus instead of SS models as in Tsuji (2008), for simplicity. We also prefer PP models rather than SS models because $\log (W / v)_{\mathrm{wk}}$ can be evaluated easily by the weighting function method for PP models (e.g. Unsöld 1955) but no such simple formula is known for SS models. A few cases we analyzed as examples are summarized in Table $4^{6}$. We proceed for each $\mathrm{M}$ giant as for Arcturus leading to Fig. 5a: We first plot observed $\log W / v$ based on the data in Table 3 (Tsuji 2008) against $\log (W / v)_{\mathrm{wk}}$ evaluated with the carbon abundance of Table 4 based on the PP model (hence differs slightly from $\log A_{C}$ in Tsuji 2008). The resulting empirical curve-of-growth is then compared with the theoretical one with $\xi_{\text {micro }}$ of Table 4 based on the PP model.

The results for CO lines in $\beta$ Peg and $\alpha$ Her are shown in Fig. 6a,b, respectively. The observed curves-of-growth of the first (filled circles) and second (filled triangles) overtones agree reasonably well with the theoretical ones for the first (solid lines) and second (dashed lines) overtones, respectively, for lines of $\log W / v \lesssim-4.75$. However, the observed data begin to deviate from the trend expected for the flat part of the curves-of-growth at $\log W / v \approx-4.75$ both in $\beta$ Peg and $\alpha$ Her. These deviations of the intermediate-strength lines are quite consistent with our LL analysis of these objects (referred to in Col. 8 of Table 4) in that these lines provided unreasonably large abundance corrections, which we have interpreted as being caused by excess absorption originating in the MOLsphere (Tsuji 2008).

The cases of $\rho$ Per and SW Vir are shown in Fig. 7a,b, respectively. In these cases, the intermediate-strength lines also show deviations from the expected theoretical curves-of-growth but in an opposite direction than for $\beta$ Peg and $\alpha$ Her (Fig. 6a,b). These results, however, are again consistent with our LL analysis of these objects (referred to in Col. 8 of Table 4), which provided much smaller abundance corrections for the intermediatestrength lines than for the weak lines, and we have interpreted these results as due to weakening of the intermediate-strength lines by the emission of the MOLsphere (Tsuji 2008).

Finally, we examine the case of the $\mathrm{OH}$ fundamental lines observed in the $L$ band region of $\mathrm{M}$ giant stars. We create curvesof-growth for $\alpha$ Her and SW Vir based on the EWs given in Table 3 (Tsuji 2008) and the results are shown in Fig. 8a,b, respectively. The observed and theoretical curves-of-growth are shown by the filled circles and solid lines, respectively. The unpredictable upturn of the observed curves-of-growth at

\footnotetext{
6 Along with the results based on PP models, those based on SS models are reproduced from Table 6 (Tsuji 2008) in Table 4 (in parenthesis). In general, the differences between PP and SS analyses do not differ significantly from the probable errors (almost the same for PP and SS analyses and not shown for SS results) of the resulting abundances and turbulent velocities.
} 
Table 4. Input data for the curves-of-growth of M giants based on PP model (results based on SS models in parenthesis).

\begin{tabular}{|c|c|c|c|c|c|c|c|}
\hline Object & Molecule & $\begin{array}{l}\text { PP model } \\
\left(T_{\text {eff }} / \log g\right)\end{array}$ & $\begin{array}{c}\xi_{\text {micro }}^{\mathrm{PP}}\left(\xi_{\text {micro }}^{\mathrm{SS}}\right) \\
\left(\mathrm{km} \mathrm{s}^{-1}\right)\end{array}$ & $\log A_{\mathrm{C}}^{\mathrm{PP}}\left(\log A_{\mathrm{C}}^{\mathrm{SS}}\right)$ & $\log A_{\mathrm{O}}^{\mathrm{PP}}\left(\log A_{\mathrm{O}}^{\mathrm{SS}}\right)$ & $\begin{array}{l}\text { CG analysis } \\
\text { (this Paper) }\end{array}$ & $\begin{array}{l}\text { LL analysis } \\
\text { (Tsuji 2008) }\end{array}$ \\
\hline$\beta \mathrm{Peg}$ & $\mathrm{CO}$ & $3600 / 0.5$ & $1.88 \pm 0.07(2.04)$ & $8.17 \pm 0.05(8.27)$ & - & Fig. 6a & Fig. 6a,b \\
\hline$\rho$ Per & $\mathrm{CO}$ & $3500 / 0.5$ & $3.52 \pm 0.73(3.59)$ & $8.17 \pm 0.08(8.27)$ & - & Fig. $7 \mathrm{a}$ & Fig. 8a,b \\
\hline$\alpha$ Her & $\mathrm{CO}$ & $3300 / 0.5$ & $2.67 \pm 0.71(2.82)$ & $8.46 \pm 0.16(8.40)$ & - & Fig. $6 b$ & Fig. 4a,b \\
\hline SW Vir & $\mathrm{CO}$ & $2900 / 0.0$ & $4.10 \pm 3.00(4.21)$ & $8.22 \pm 0.22(8.26)$ & - & Fig. 7b & Fig. 9c \\
\hline$\alpha$ Her & $\mathrm{OH}$ & $3300 / 0.5$ & $2.57 \pm 0.09(2.92)$ & - & $8.82 \pm 0.05(8.85)$ & Fig. $8 \mathrm{a}$ & Fig. 10a,b \\
\hline SW Vir & $\mathrm{OH}$ & $2900 / 0.0$ & $1.69 \pm 0.15(1.83)$ & - & $8.42 \pm 0.12(8.54)$ & Fig. 8b & Fig. 11c \\
\hline
\end{tabular}

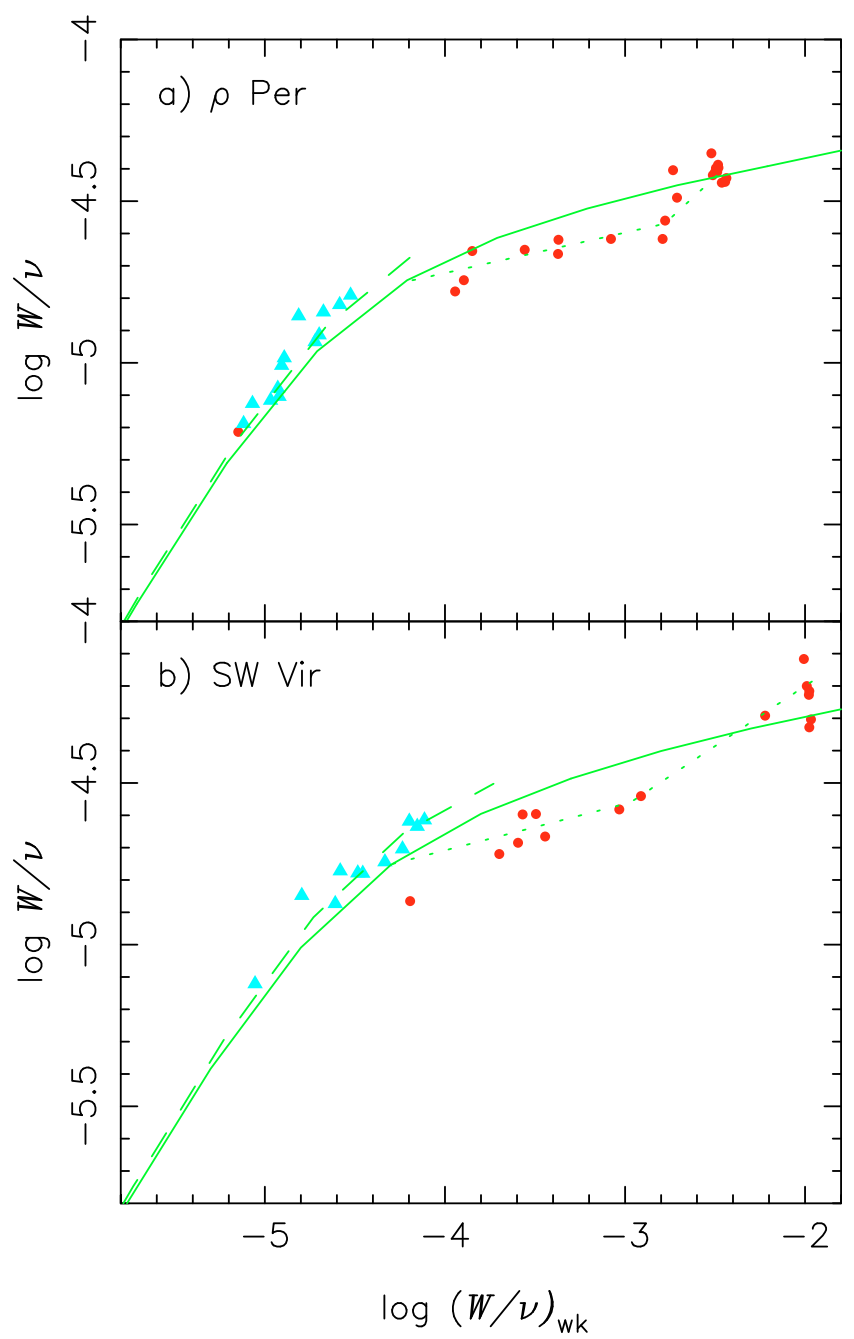

Fig. 7. The same as Fig. 6 but note that the intermediate-strength lines show equivalent widths smaller than those predicted by the theoretical curves-of-growth. a) $\rho$ Per (M4II). b) SW Vir (M7III:).

$\log W / v \approx-4.75$ appears more clearly for $\mathrm{OH}$ than for $\mathrm{CO}$ discussed above. This is simply because $\mathrm{OH}$ data are superior to $\mathrm{CO}$ data both in quality and quantity. Thus, the peculiar behaviors of the intermediate-strength lines noted by our LL analysis (referred to in Col. 8 of Table 4) can be shown more simply by the $\mathrm{CG}$ analysis.

Based on the classical theory of line formation, the theory of curve-of-growth is well established (e.g. Unsöld 1955). However, such an upturn (or downfall) in the flat part of the curves-of-growth identified in this subsection is quite unexpected, yet important to clarifying its origin. There is probably little possibility of explaining these effects in the flat part of the curves-of-growth within the framework of the theory of line formation in homogeneous photospheres. For example, for the low densities in the photospheres of giant stars, the upturn cannot be attributed to the development of the damping wings. Microturbulence would also not exhibit such an abrupt upturn as observed. For this reason, we propose that it is reasonable to interpret the unusual pattern of the curves-of-growth as evidence for the hybrid nature of the infrared spectra.

\subsection{Red supergiant stars}

Because the CG method has been applied more often in the past, we can examine whether the anomalous behaviors of the intermediate-strength lines can be found in the curves-of-growth published by other authors. First, we recall that Lambert et al. (1984) showed in their Fig. 2 a curve-of-growth of $\mathrm{OH}$ fundamental lines for M2 supergiant Betelgeuse and noted that the stronger lines cannot be fitted by the theoretical curve-of-growth. We note that the behavior of the stronger $\mathrm{OH}$ lines in Betelgeuse is quite similar to that found in Arcturus as well as M giant stars. Inspection of their Fig. 2 indicates that the upturn in the stronger $\mathrm{OH}$ lines also starts at about $\log W / v \approx-4.75$ or at slightly weaker lines, and the deviation from the flat part of the theoretical curve-of-growth is more significant in this supergiant star than in the red giant stars that we have studied.

Several possible origins of this unusual behavior of strong $\mathrm{OH}$ lines (or the intermediate-strength and the strong lines in our classification) in Betelgeuse were considered by Lambert et al. (1984), although the problem remains unexplored since then as far as we are aware. Because of the similarity between the phenomenon in Betelgeuse and that in M giant stars, it is natural to interpret the magnificent upturn of the curve-of-growth of Betelgeuse as being caused by an extensive MOLsphere in this red supergiant star.

\subsection{Mira-type variables}

The CG method has been extensively applied to the infrared molecular lines in Mira-type variables by Hinkle and his colleagues. First, Hinkle (1978) showed that the spectra of Mira variables are quite complicated in that a molecular line generally consists of multiple components with different temperatures and velocities. The CG method was extended to the $\mathrm{H}_{2} \mathrm{O}$ ro-vibrational lines in R Leo by Hinkle \& Barnes (1979), who found that two separate layers, warm and cool, contribute to the $\mathrm{H}_{2} \mathrm{O}$ spectrum. Their curve-of-growth for the warm-component lines (their Fig. 4) shows an upturn at $\log W / v \approx-4.75$ or at slightly weaker lines, and the observed curve-of-growth for the cool-component lines (their Fig. 6) shows more clearly that the 


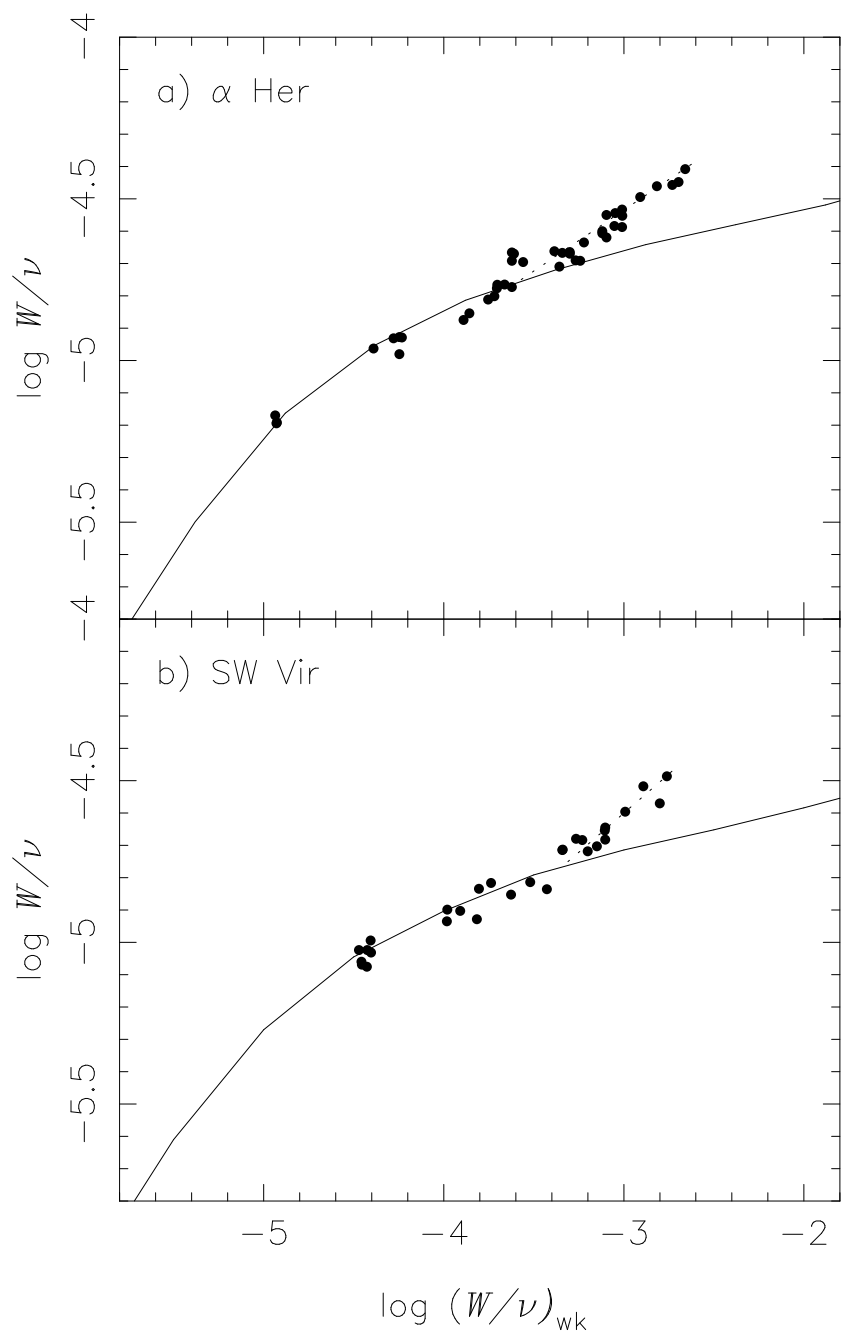

Fig. 8. Empirical curves-of-growth of the $\mathrm{OH}$ fundamentals (filled circles) are compared with the theoretical ones (solid lines) based on the PP models and the microturbulent velocities given in Table 4 . Note that the intermediate-strength lines show systematically larger equivalent widths than those predicted by the theoretical curves-of-growth. a) $\alpha$ Her (M5Ib-II). b) SW Vir (M7III:).

strong lines deviate significantly from the flat part of the theoretical curve-of-growth.

A time series of 32 high resolution FTS spectra of another Mira variable $\chi$ Cyg was analyzed by Hinkle et al. (1982), who clarified the detail of the Mira variability including the photospheric pulsation, shock generation, and circumstellar structure with an $800 \mathrm{~K}$ stationary layer discovered by them. We consider here only their CO curves-of-growth of $\chi$ Cyg shown in their Fig. 4: the curves-of-growth show significant changes in the shape of the strong line portion with phase and their gradients are often too steep to be fitted to the flat part of the curve-ofgrowth expected for the low gravity atmospheres typical of Mira variables. The gradients in some phases are indeed as steep as those expected for the damping part of the curve-of-growth. We conclude that the peculiar shapes of the curves-of-growth in the strong line portion are rather general features in Mira variables.

The anomalous shapes of the curves-of-growth in Mira variable stars are not necessarily associated with special problems of variability, since more or less similar phenomena are found in ordinary giants including Arcturus and many $\mathbf{M}$ giants, and
Table 5. Differential line-shifts between low $(\mathrm{L})$ and high $(\mathrm{H})$ excitation lines in radial velocities measured at the peak absorption $(\mathrm{P})$ and at the mid-point of $F W H M(\mathrm{M})$. ME is the mean error of the radial velocity.

\begin{tabular}{ccccc}
\hline \hline CO band & $\begin{array}{c}V_{\mathrm{P}}^{\mathrm{H}}-V_{\mathrm{P}}^{\mathrm{L}}(\mathrm{ME}) \\
\left(\mathrm{km} \mathrm{s}^{-1}\right)\end{array}$ & $\begin{array}{c}V_{\mathrm{M}}^{\mathrm{H}}-V_{\mathrm{M}}^{\mathrm{L}}(\mathrm{ME}) \\
\left(\mathrm{km} \mathrm{s}^{-1}\right)\end{array}$ & $N$ & $\begin{array}{c}\text { sp. ref. } \\
(\text { Table 1) }\end{array}$ \\
\hline$\Delta v=1$ & $0.61(0.08)$ & $0.45(0.07)$ & 24 & 1 \\
& $0.31(0.07)$ & $0.52(0.09)$ & 17 & 2 \\
& $0.28(0.09)$ & $0.37(0.10)$ & 13 & 3 \\
& $0.27(0.08)$ & $0.24(0.09)$ & 13 & 4 \\
& $0.26(0.07)$ & $0.35(0.06)$ & 22 & 5 \\
$\Delta v=2$ & $0.08(0.03)$ & $0.18(0.04)$ & 27 & 6 \\
$\Delta v=3$ & $0.09(0.01)$ & $0.07(0.01)$ & 117 & 7 \\
\hline
\end{tabular}

should reflect some fundamental problems of line formation in cool luminous stars in general.

\section{Line intensities, shifts, and shapes}

We so far discussed mainly equivalent widths and found that the strong lines show unusual behaviors difficult to explain by the classical theory of line formation. We further examine whether there is any peculiarity in the observed characteristics of the stronger lines compared to the weaker lines in Arcturus.

\subsection{Line intensities}

We plot the values of $\log W / v$ against the lower excitation potentials (LEPs) for the lines of the CO first overtone and fundamental bands observed in Arcturus in Fig. 9a,b, respectively. The dotted lines show the critical value of $\log W / v \approx-4.75$. It is immediately clear that the lines stronger than the critical value are limited to lines with LEP lower than about $1.3 \mathrm{eV}$ (overtone bands) or $1.5 \mathrm{eV}$ (fundamental bands). This means that the excess absorption in Arcturus should originate in the region of low excitation but not necessarily be very low.

\subsection{Line shifts}

We measure the shifts of individual lines and obtain the corresponding radial velocities for them. The different spectral regions were observed at different epochs (see Table 1) and it is difficult to convert the radial velocities to a consistent absolute scale accurately because the reference laser and signal beams do not necessarily pass through the FTS on parallel paths (Hinkle et al. 1995). However, we do not need to know the radial velocities themselves for our purposes and we measure differential line-shifts between high and low excitation lines in each spectrum. We refer to the radial velocity of a line as $V^{\mathrm{H}}$ or $V^{\mathrm{L}}$ according to whether its LEP is higher or lower than $10000 \mathrm{~cm}^{-1}$ $(\approx 1.25 \mathrm{eV})$. We also measure the shifts of a line at its peak absorption and mid-point of $F W H M$, and distinguish them by suffixes $\mathrm{P}$ and $\mathrm{M}$, respectively.

The resulting mean values of the differential line shifts in the peak position and the mid-point of $F W H M$ are given in Cols. 2 and 3, respectively, of Table 5 with the mean errors (ME) of the radial velocities in parenthesis. The number of lines measured is given in Col. 4 and the identification number of the spectrum referred to in Table 1 is given in Col. 5. Inspection of Table 5 reveals that all the spectra exhibit positive differential shifts in $V^{\mathrm{H}}-V^{\mathrm{L}}$, indicating that the low excitation lines (largely the intermediate-strength lines) are expanding slightly compared to 


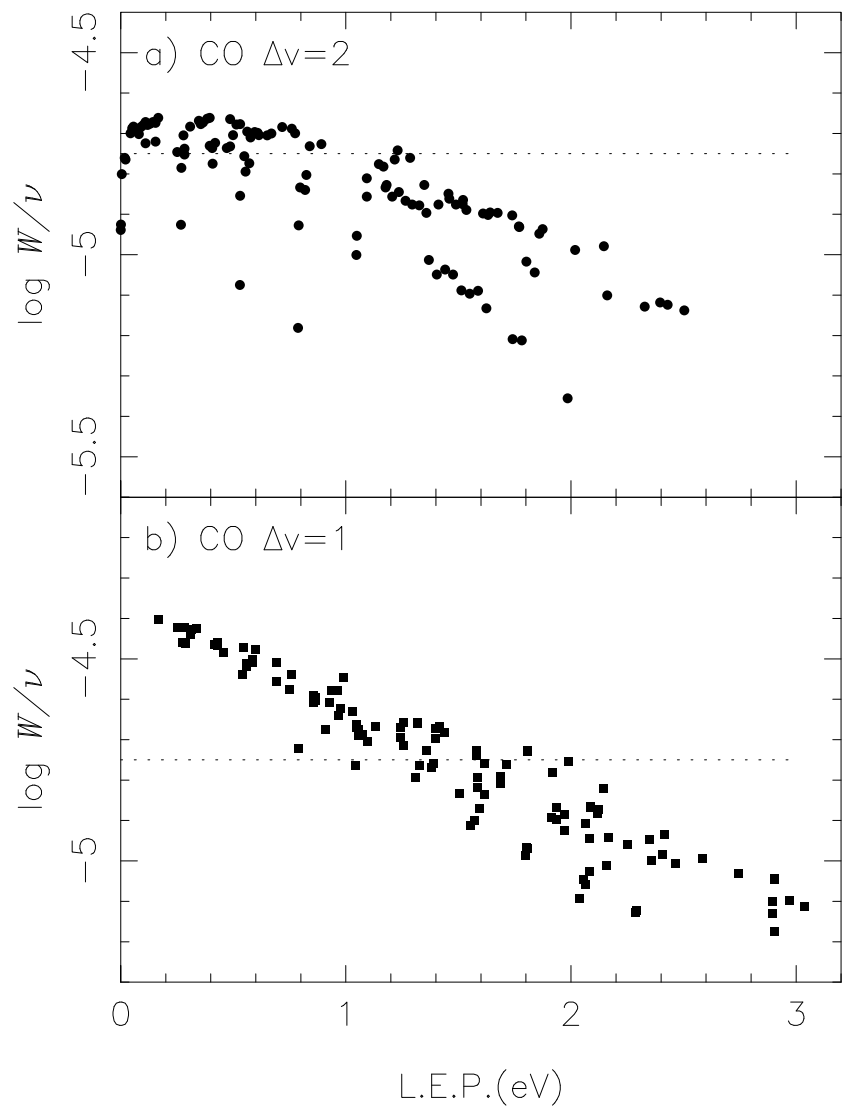

Fig. 9. The values of $\log W / v$ of $\mathrm{CO}$ lines observed in Arcturus are plotted against the lower excitation potential (L.E.P). The critical value of $\log W / v \approx-4.75$ is indicated by the dotted lines. a) The CO first overtone bands. b) The CO fundamental bands.

the high excitation lines (mostly the weak lines). The differential shifts, however, are smaller in the overtone bands than in the fundamental bands.

We also examine the line asymmetry defined by $V_{\mathrm{P}}-V_{\mathrm{M}}$ and the results are plotted against LEP in Fig. 10a-d, separately for different $\mathrm{CO}$ bands ( $\mathrm{a} \& \mathrm{~b}$ : fundamentals by winter \& summer data, respectively, c: first overtones, d: second overtones). The solid lines represent linear fits to the data and it appears that the line asymmetry depends little on LEP. The mean values of $V_{\mathrm{P}}-V_{\mathrm{M}}$ are $0.00,-0.08,-0.09$, and -0.12 for $\mathrm{a}, \mathrm{b}, \mathrm{c}$, and $\mathrm{d}$, respectively. The result that the mean values of $V_{P}-V_{M}$ tend to be negative agrees well with our previous measurements for Arcturus as well as for many M-giants (Tsuji 1991). Our analysis can also be regarded as a highly simplified version of the bisector analysis, a result of which on Arcturus for Fe I lines by Dravins (1987) indicates that $V_{\mathrm{P}}-V_{\mathrm{M}}$ should be negative from his Fig. 1. We conclude that $\mathrm{CO}$ lines including those of fundamental bands show only minor asymmetry if any.

\subsection{Line broadening}

We measure FWHMs and line-depths, and the results are given in Table 2. The smoothing was applied to the atlas spectra and we correct its effect on $F W H M$ s with the Gaussian of $F W H M=$ $1.25 \times$ Res (Hinkle et al. 1995). The resulting intrinsic $F W H M$ is converted to the Doppler velocity dispersion $\xi_{\mathrm{D}}$ by $F W H M=$ $2 \sqrt{\operatorname{Ln} 2}(v / c) \xi_{\mathrm{D}}$. The resulting values of $\xi_{\mathrm{D}}$ are plotted in Fig. 11 against the intrinsic line intensities represented by $\log (W / v)_{\mathrm{wk}}$ defined by Eq. (1). It is to be noted in Fig. 11 that the lines

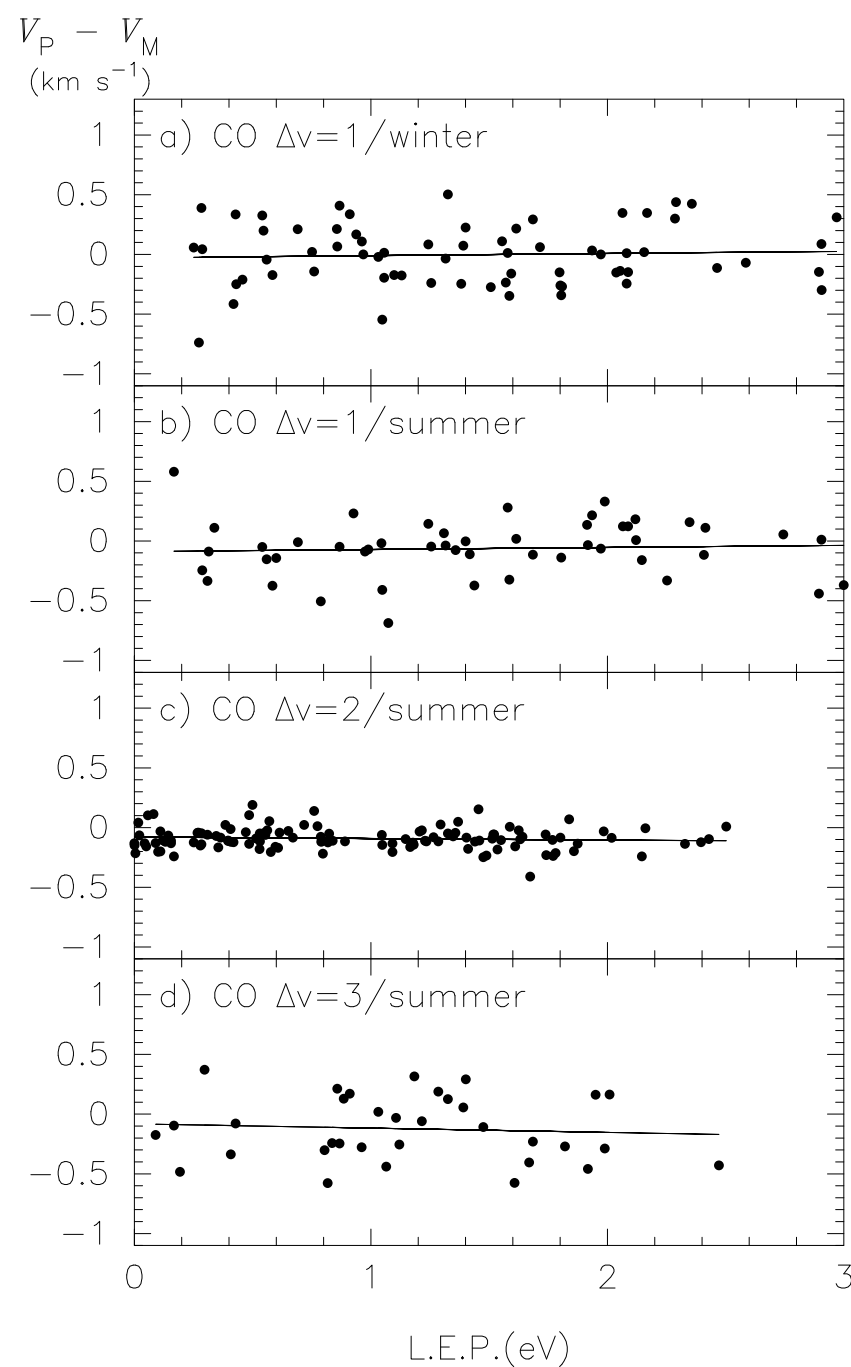

Fig. 10. The line asymmetries defined by $V_{\mathrm{P}}-V_{\mathrm{M}}$, where $V_{\mathrm{P}}$ and $V_{\mathrm{M}}$ are the radial velocities at the peak of absorption and at the mid-point of the half-maximum intensities, respectively, are plotted against LEP (in eV). The solid lines represent linear fits to the data. a) The CO fundamental bands (winter data: sp. Refs. 1-4 in Table 1). b) The CO fundamental bands (summer data: sp. Refs. 5, 6). c) The CO first overtone bands (summer data: sp. Ref. 7). d) The CO second overtone bands (summer data: sp. Ref. 7).

of CO fundamentals, first, and second overtones shown by the filled squares, circles, and triangles, respectively, form a continuous curve. At first, we plotted observed $\xi_{\mathrm{D}}$ values against measured line-depths. However, plots of the overtone and fundamental lines formed separate curves rather than a unified curve such as in Fig. 11, and this may be because the line-depth cannot be a good measure of intrinsic line intensity especially if applied to different spectral regions.

We assume that the lines of the $\mathrm{CO}$ second overtones and relatively weak lines of the first overtones should originate in the photosphere from our analysis outlined in Sect. 3.1. The line-widths of these lines then can be interpreted as being photospheric in origin. We extrapolate the measured Doppler velocities to the weak-line limit in Fig. 11 and find the limiting $\xi_{\mathrm{D}}$ value of $4.07 \mathrm{~km} \mathrm{~s}^{-1}$. This result cannot be explained by the microturbulent velocity of $1.87 \mathrm{~km} \mathrm{~s}^{-1}$ and thermal velocity dispersion of $\xi_{\text {th }} \approx 1 \mathrm{~km} \mathrm{~s}^{-1}$. We conclude that an additional 


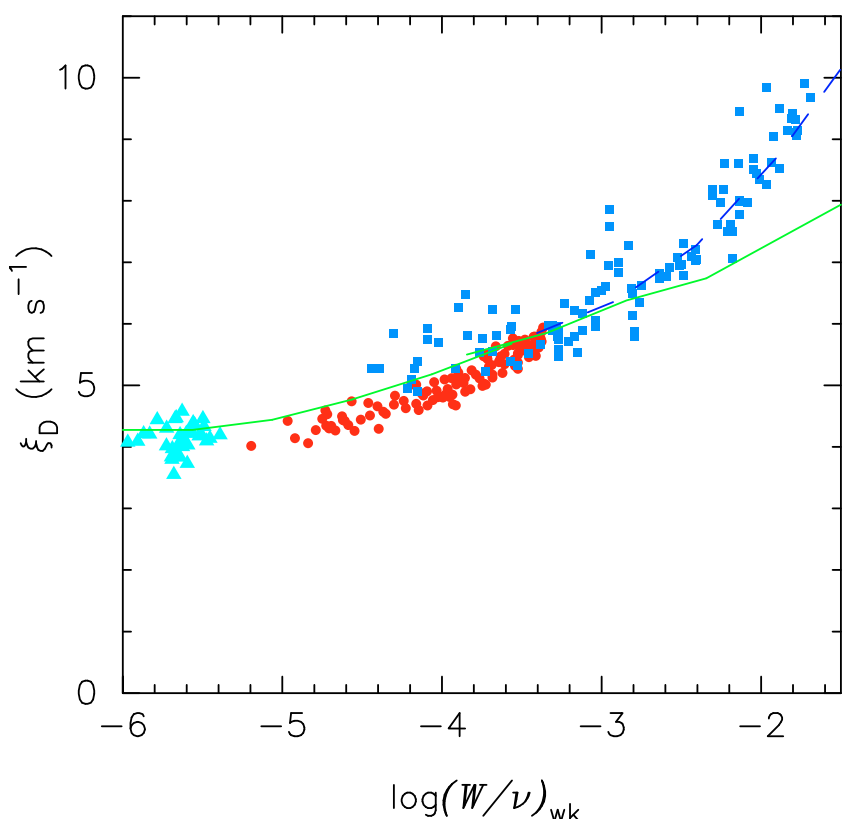

Fig. 11. The Doppler velocity dispersions $\xi_{\mathrm{D}}\left(\right.$ in $\left.\mathrm{km} \mathrm{s}^{-1}\right)$ of the CO lines observed in Arcturus are plotted against $\log (W / v)_{\text {wk }}$, which can be a measure of the intrinsic line intensity. The CO fundamental, first, and second overtone bands are represented by the filled squares, circles, and triangles, respectively. The predicted $\xi_{\mathrm{D}}$ values based on the photospheric model with $\xi_{\text {micro }}=1.87 \mathrm{~km} \mathrm{~s}^{-1}$ and $\xi_{\text {macro }}=3.47 \mathrm{~km} \mathrm{~s}^{-1}$ are shown by the solid lines. The effect of molecular clouds with $\xi_{\text {micro }}=4.0 \mathrm{~km} \mathrm{~s}^{-1}$ in our ad-hoc model is shown by the dashed line (as for detail, see Sect. 6.2).

broadening with velocity dispersion of $\left(\xi_{\mathrm{D}}^{2}-\xi_{\text {th }}^{2}-\xi_{\text {micro }}^{2}\right)^{1 / 2} \approx$ $3.47 \mathrm{~km} \mathrm{~s}^{-1}$ is required and we attribute its origin to large-scale photospheric motions, which are referred to as macroturbulence.

It is by no means clear, however, if all the results shown in Fig. 11 can be understood with the micro and macroturbulent velocities noted above. We examine this problem by evaluating the Doppler velocities for the fictitious lines used to generate the theoretical curves-of-growth in Sect. 4.1. To each computed line profile based on the photospheric model (model a in Fig. 2) with $\xi_{\text {micro }}=1.87 \mathrm{~km} \mathrm{~s}^{-1}$ throughout the photosphere, we apply the broadening caused by macroturbulence with velocity dispersion of $\xi_{\text {macro }}=3.47 \mathrm{~km} \mathrm{~s}^{-1}$, and measure the Doppler velocity as we did for the observed spectra. The results are shown in Fig. 11 by the solid lines, of which the weak and strong line parts are based on the fictitious lines of the $\mathrm{CO}$ first overtones and fundamentals, respectively. The weak and strong line parts join at $\log (W / v)_{\mathrm{wk}} \approx-3.5$. The predicted and observed Doppler velocities roughly agree for most lines with $\log (W / v)_{\mathrm{wk}} \lessgtr-3.0$, although further fine tuning in the thermal and/or velocity structures should be required to have a perfect fit.

We know that the lines of the $\mathrm{CO}$ fundamentals do not originate in the photosphere alone. The observed Doppler velocities in fact appear to be much higher than the predicted ones for the stronger $\mathrm{CO}$ lines with $\log (W / v)_{\mathrm{wk}}>-3.0$ in Fig. 11. Clearly, the stronger $\mathrm{CO}$ lines require additional broadening other than the micro and macroturbulence so far considered. The stronger lines of the $\mathrm{CO}$ fundamentals, which can be explained neither by the RE photospheric model nor by the hot chromospheric model (Fig. 5b), are showing evidence for a cool constituent responsible to the strong absorption. The additional broadening of the stronger fundamental lines shown in Fig. 11 is consistent with this conclusion from our CG analysis, and the cool constituent responsible for additional absorption should be related to significantly large turbulence (further discussed in Sect. 6.2).

\section{Discussion}

Some observational data for Arcturus and other cool luminous stars cannot be explained by our present understanding of stellar atmospheres. Although we have no solution on this difficulty at present, we examine some possibilities.

\subsection{The case of Arcturus}

A difficulty in the spectrum of Arcturus was highlighted by the detailed line-by-line analysis, which exhibited excess absorption in the lines of $\log W / v>-4.75$ (Figs. 3 and 4). The specific lines that show excess absorption originate in levels of relatively low excitation potentials but as high as $1 \mathrm{eV}$ or higher (Fig. 9). This fact implies that the excess absorption should be formed in an environment at relatively cool but not extremely cool. The problem is also evident in the curves-of-growth, which show an unexpected upturn in their flat part at about $\log W / v \approx-4.75$ (Fig. 5). This sudden upturn is difficult to understand within the framework of the line formation theory in a homogeneous photosphere, and a possible explanation may require an additional constituent other than the hot chromosphere, which tends to weaken rather than to strengthen the $\mathrm{CO}$ lines.

In the outer atmosphere of Arcturus, the possible presence of a cool component referred to as a CO-mosphere was suggested to explain the strong CO lines by Wiedemann et al. (1994), who considered a thermal bifurcation model consisting of hot and cool components. The cool component was proposed to be generated by the molecular cooling caused by $\mathrm{CO}$ and other molecules. This molecular cooling was, however, already included in constructing the RE model photosphere and, for this reason, the surface temperature of our model employed in this study is as low as $\approx 2300 \mathrm{~K}$ at $\log \tau_{0}=10^{-6}$ (Fig. 2). However, as we know already, this RE model photosphere of very low surface temperature could not explain the excess absorption of the strong CO fundamental lines at all (Figs. 4 and 5b).

For the reason outlined above, we propose that an additional constituent other than the cool and hot components shown in Fig. 2 should be introduced. The presence of such a third constituent would be consistent with the large Doppler velocities of the strong CO fundamental lines as noted in Fig. 11. However, the strong $\mathrm{CO}$ fundamental lines appear to exhibit only minor line-asymmetry and only small outflow motion relative to the $\mathrm{CO}$ weaker lines that possibly originate in the photosphere, as shown in Sect. 5.2.

From the empirical data summarized above, we suggest that molecular condensations may be formed within the atmosphere of Arcturus. The molecular condensations may not necessarily form a separate structure but may be floating in the atmosphere as molecular clouds. These molecular clouds then show only small motions relative to the surrounding atmosphere from which the clouds are formed, and the CO lines should not necessarily exhibit asymmetry nor high outflow velocity. The turbulent velocities in the clouds, however, can be high due to possible dynamical effects associated with the cloud formation, and the large Doppler velocities of the strong CO fundamental lines (Fig. 11) can be consistent with this scenario.

A problem is how the additional material needed for cloud formation can be made available and how the molecular clouds can be formed. For this purpose, radiation and turbulent 
pressures are insufficient, as is clear from the fact that extended hydrostatic models included such effects resulted in only very low density extended photosphere (Sect. 2.4 and Tsuji 2006). One possibility is that the atmospheres of red giant stars are not so clear as assumed in the classical RE model photospheres but may contain some additional material supplied by surface activities of various kinds (Sect. 6.4). Once density inhomogeneity appears in the huge atmosphere of red giant stars, it may grow further by the thermal instability triggered by the cooling due to the strong infrared radiation by molecular bands as suggested, for example, by Muchmore et al. (1987). The molecular cooling has already been included in the hydrostatic RE model photospheres, but this mechanism can play a more important role in cloud formation if applied to the gaseous material in free space. Cuntz \& Muchmore (1994) demonstrated that domains of radiative instabilities caused by $\mathrm{CO}$ and $\mathrm{SiO}$ cooling exist within the outer atmospheres of red (super)giant stars, and molecular clouds can be formed in these instability islands.

\subsection{An ad-hoc model}

We propose the presence of molecular clouds in the atmosphere of Arcturus to explain the available observed data presented in Sects. 3-5. At present, we have no precise idea about the location, temperature, or density of the clouds. However, we now try some numerical exercises to have some assessments on the nature of the clouds by considering an ad-hoc model. We first assume that the clouds may be formed in the upper atmosphere where strong lines are formed and temperatures can in general be low. Also, if many clouds are formed to have definite observational effects, the net effects of them can be approximated by a shell consisting of many overlapping clouds, and we apply the MOLsphere model that we assumed in the case of red supergiant stars (Tsuji 2006). This does not necessarily imply that a separate "sphere" exists in the outer part of the atmosphere but we use this model only for convenience in numerical analysis.

We start from an extended photosphere in radiative and hydrodynamical equilibria (SS model) starting integration from $\log \tau_{0}=-9.0$ (model $b$ in Fig. 2). The geometrical extension $R_{\text {out }}$ depends on the turbulent pressure and we assume a turbulent velocity $\xi_{\mathrm{Mol}}$ as a free parameter. Our extended RE model with $\xi_{\mathrm{Mol}}=6 \mathrm{~km} \mathrm{~s}^{-1}\left(\log \tau_{0}=-9.0\right.$, model b in Fig. 2) produced $R_{\text {out }}=28.33 R_{\odot}=1.11 R_{*}$. We now make ad-hoc assumptions of a uniform temperature of $T_{\mathrm{Mol}}$ and a column density of $N_{\text {Mol }}$ in the layers above a critical depth of $\log \tau_{0}^{\text {cr }}$ at a geometrical radius $R_{\text {in }}$. The resulting model is in neither radiative nor hydrostatic equilibrium. Then, we take $\log \tau_{0}^{\mathrm{cr}}=-6.0$ where $R_{\text {in }}=26.57 R_{\odot}=1.04 R_{*}$. We note that this model is essentially identical to introducing a shell of given inner and outer radii, turbulent velocity, temperature, and density. We use this ad-hoc model simply because our spectral synthesis code can be applied directly to such a model and the photosphere can be included automatically as a boundary condition.

We try several combinations of $T_{\mathrm{Mol}}$ and $N_{\mathrm{Mol}}$ for the shell with inner and outer boundaries at $1.04 R_{*}$ and $1.11 R_{*}$, respectively. We have assumed the turbulent velocity of $6 \mathrm{~km} \mathrm{~s}^{-1}$ in modeling the extended photosphere, but this was simply as a means by which to generate our ad-hoc model based on the extended RE model. We must determine microturbulent velocity $\xi_{\text {micro }}^{\mathrm{Mol}}$ in the MOLsphere anew to be consistent with observations. For this purpose, we try several values for $\xi_{\text {micro }}^{\mathrm{Mol}}$ and examine if the observed Doppler velocities of the strong CO lines in Fig. 11 can be reproduced with our fictitious lines of the $\mathrm{CO}$

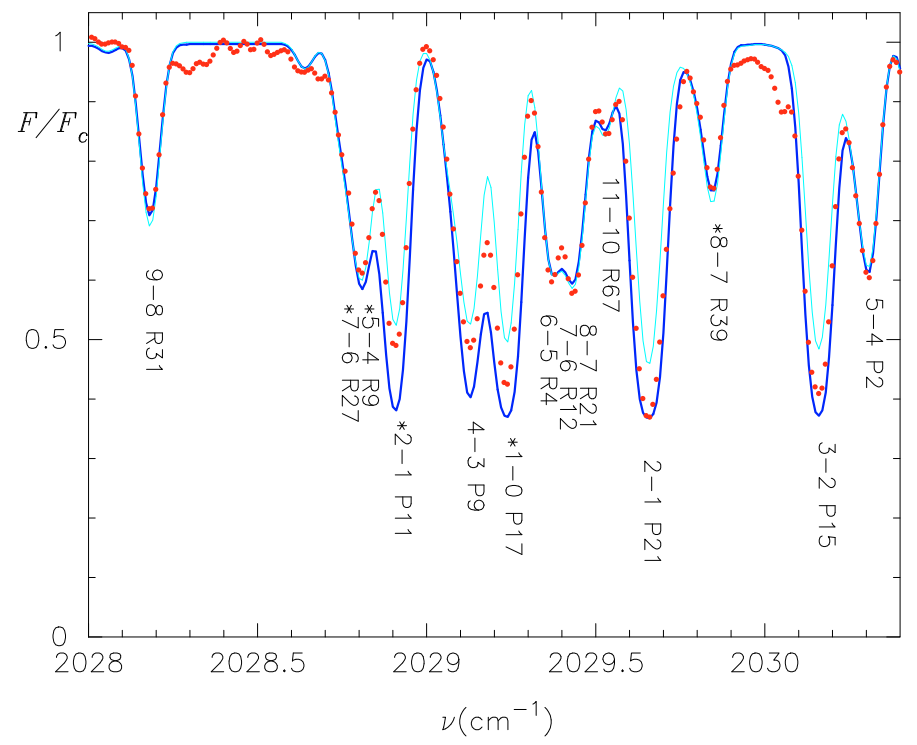

Fig. 12. Predicted spectra based on the RE model photosphere (thin line) and the ad-hoc model discussed in the text (thick line) are compared with the observed spectrum of Arcturus (filled circles). The line identifications given by Hinkle et al. (1995) are reproduced: ${ }^{13} \mathrm{C}^{16} \mathrm{O}$ lines are marked with asterisks and ${ }^{12} \mathrm{C}^{16} \mathrm{O}$ lines without.

fundamentals used in Sect. 5.3. This analysis is done iteratively with that of the observed spectrum of $\mathrm{CO}$ fundamentals represented by Fig. 12 (filled circles). For this purpose, we now compute a synthetic spectrum of $\mathrm{CO}$ fundamentals corresponding to Fig. 12 by the use of our ad-hoc model with $\xi_{\text {micro }}^{\text {Mol }}$ in the MOLsphere and $\xi_{\text {micro }}=1.87 \mathrm{~km} \mathrm{~s}^{-1}$ in the RE photosphere included as the boundary condition. Some lines of ${ }^{13} \mathrm{CO}$ are included and we assume a ${ }^{12} \mathrm{C} /{ }^{13} \mathrm{C}$ ratio of 7 (Hinkle et al. 1976). The resultant synthetic spectrum is convolved with the smoothing function of the atlas (Gaussian with $F W H M=1.25 \times$ Res) and the macroturbulent broadening of the velocity dispersion $\xi_{\text {macro }}=3.47 \mathrm{~km} \mathrm{~s}^{-1}$ (Sect. 5.3).

The results of our trial and error are shown in Fig. 11 for the Doppler velocity and in Fig. 12 for the synthetic spectrum. The parameters that provide reasonable fits are found to be: $\xi_{\text {micro }}^{\text {Mol }}=4.0 \mathrm{~km} \mathrm{~s}^{-1}, N_{\mathrm{Mol}}(\mathrm{CO})=5 \times 10^{+19} \mathrm{~cm}^{-2} 7$, and $T_{\text {Mol }}=2000 \mathrm{~K}$. With these parameters, the large Doppler velocities of the stronger $\mathrm{CO}$ lines are reproduced as shown by the dashed line in Fig. 11. As shown in Fig. 12, the observed strong CO lines (filled circles) can be reproduced approximately by the prediction based on our ad-hoc model (thick line), but are too deep to be accounted for by the predicted spectrum based on the RE model photosphere alone (thin line). On the other hand, the observed weaker CO lines (e.g. 9-8 R31, *8-7 R39) are slightly shallower than the predictions based on the RE photospheric model (thin line), in a way consistent with the CG analysis (Fig. 5b), but can be fitted by the predictions based on our ad-hoc model (thick line).

We also compute theoretical curve-of growth for our adhoc model with the same parameters used in the computation of the synthetic spectrum and the Doppler velocity. The result is shown by the solid line in Fig. 13. For comparison, we reproduce the predicted CG for the RE photospheric model (dotted lines)

\footnotetext{
7 With the $\mathrm{C} / \mathrm{H}$ ratio of $10^{-4}$, this $\mathrm{CO}$ column density implies $\mathrm{H}$ column density of $5 \times 10^{+23} \mathrm{~cm}^{-2}$ or mass column density of about $1 \mathrm{~g} \mathrm{~cm}^{-2}$, which is about the same as that above the temperature minimum in the empirical chromospheric model (Ayres \& Linsky 1975).
} 


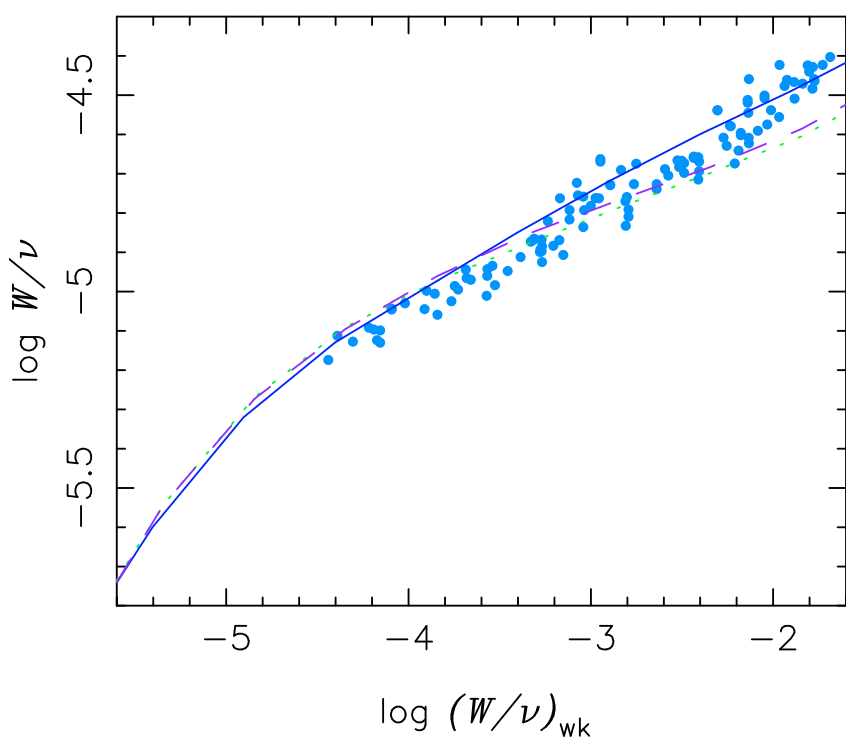

Fig. 13. Predicted theoretical curve-of-growth for our ad-hoc model (solid line) is compared with the observed data on $\mathrm{CO}$ fundamental lines (filled circles) and the theoretical curve-of-growth for the RE photospheric model (dotted line) reproduced from Fig. 5b. Predicted theoretical curve-of-growth for another ad-hoc model in which temperatures in the surface layers are reduced by as much as $250 \mathrm{~K}$ is shown by the dashed line (as for detail, see the text).

together with the observed data (filled circles) from Fig. 5b. Now, the basic features of the observed data are that the relatively weak lines are below the theoretical CG for RE photospheric model and the stronger lines are above it, which can roughly be accounted for by the theoretical CG including the effect of molecular clouds (solid line). The fits, however, are not so good for lines around $\log W / v \approx-5.0$ and further refinement of our model is needed.

For the result that the weaker $\mathrm{CO}$ fundamental lines tend to be below the theoretical curve-of-growth based on the RE photospheric model (Fig. 5b), we recalled in Sect. 4.1 the possibility that the EWs can be reduced slightly by the emission caused by the chromospheric temperature inversion (Heaseley et al. 1978). An alternative possibility to resolve this issue, however, is that the reduction in EWs may be caused by the thermal emission of the molecular clouds as shown in Fig. 12. We prefer the possibility of molecular clouds rather than chrompsphere, because the molecular cloud model provides a consistent interpretation of the weaker and stronger CO fundamental lines, while the chromospheric effect is supposed to be rather minor in Arcturus if present at all (Wiedemann et al. 1994).

The case discussed above is probably neither the optimal nor unique solution, but we show only an example of how the observed deep cores of the $\mathrm{CO}$ fundamental lines can be accounted for with the molecular clouds, whose total $\mathrm{CO}$ column density is about $5 \times 10^{+19} \mathrm{~cm}^{-2}$. We do not think that it is useful to explore such an ad-hoc model in more detail. We note, however, that the extension of the shell cannot be very large, since strong $\mathrm{CO}$ lines will appear as emission lines for $R_{\text {in }}$ and/or $R_{\text {out }}$ larger than those assumed above (the weaker lines already contribute to emission as noted in the preceding paragraph $)^{8}$, so long as we assume a spherically symmetric and homogeneous shell.

\footnotetext{
8 This example that the molecular clouds produce either emission or absorption depending on the line-strengths (and possibly on other parameters that define the shell) lends a supporting argument for our interpretation of either strengthening (e.g. $\beta$ Peg and $\alpha$ Her in Fig. 6) or weakening (e.g. $\rho$ Per and SW Vir in Fig. 7) of the intermediate-strength
}

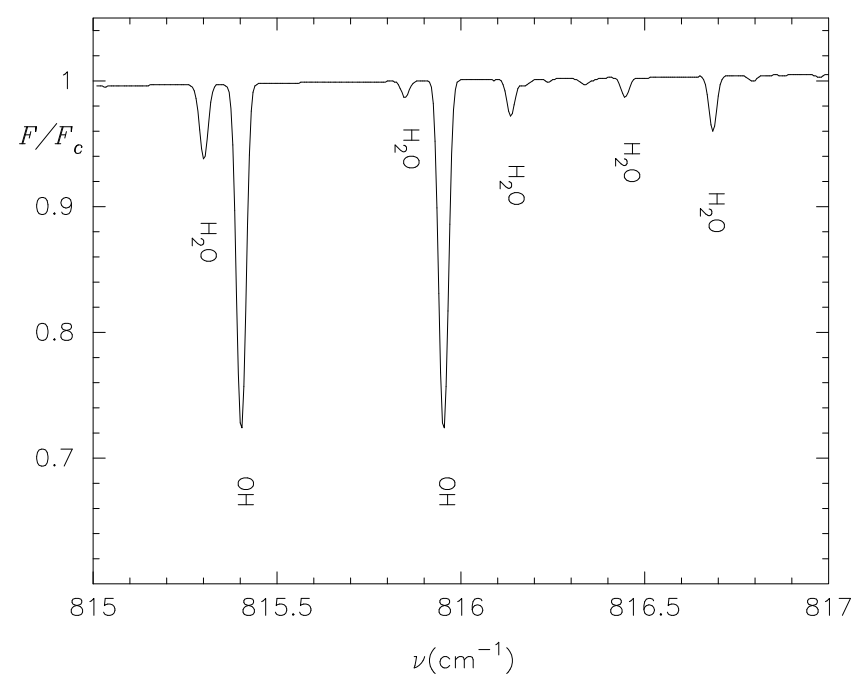

Fig. 14. Predicted spectrum of the pure rotation lines of $\mathrm{OH}$ and $\mathrm{H}_{2} \mathrm{O}$ based on an ad-hoc model discussed in the text. $\mathrm{OH}$ lines are mostly formed in the photosphere while $\mathrm{H}_{2} \mathrm{O}$ lines in the molecular clouds or the MOLsphere. As to the observed spectrum of Arcturus, see Fig. 1 of Ryde et al. (2002).

We also examine whether the unexpected detection of pure rotation lines of $\mathrm{H}_{2} \mathrm{O}$ by Ryde et al. (2002) in Arcturus can be interpreted in the same way. For this purpose, we retain all the features in our ad-hoc model discussed above except for the column density. We assume again the microturbulent velocities of $\xi_{\text {micro }}^{\text {Mol }}=4.0 \mathrm{~km} \mathrm{~s}^{-1}$ in the MOLsphere and $\xi_{\text {micro }}=1.87 \mathrm{~km} \mathrm{~s}^{-1}$ in the photosphere, and apply linelists of $\mathrm{H}_{2} \mathrm{O}$ (Partridge \& Schwenke 1997) and OH (Jacquinet-Husson et al. 1999). An example of the predicted spectrum with $N_{\mathrm{Mol}}\left(\mathrm{H}_{2} \mathrm{O}\right)=2 \times$ $10^{+17} \mathrm{~cm}^{-2}$ is shown by the solid line in Fig. 14. The observed $\mathrm{H}_{2} \mathrm{O}$ lines (Fig. 1 of Ryde et al. 2002) can be accounted for roughly by our ad-hoc model.

This is certainly not a unique solution: For example, Ryde et al. (2002) showed that their observation of $\mathrm{H}_{2} \mathrm{O}$ lines in Arcturus can be explained if the surface temperatures of the RE model can be lowered by about $300 \mathrm{~K}$. A possibility of such a surface cooling was predicted by non-LTE models of Arcturus (Short \& Hauschildt 2003). The largest effect was shown for a plane-parallel case including $\mathrm{Fe}$, Ti, and light elements in NLTE, and the surface temperature was lowered by about $250 \mathrm{~K}$ compared to the corresponding LTE model. We introduce the lowerings beginning with $250 \mathrm{~K}$ at the surface, as shown in their Fig. 1, into our LTE model, and solve chemical and hydrostatic equilibria for the modified temperature structure. We use the resulting model to computing $\mathrm{CO}$ lines and construct curve-of-growth as we did for our ad-hoc model. We assume microturbulent velocity of $1.87 \mathrm{~km} \mathrm{~s}^{-1}$ throughout the photosphere as in our original photospheric model. The result is shown by the dashed line in Fig. 13.

Inspection of Fig. 13 reveals that the $\mathrm{CO}$ lines can also be strengthened somewhat by the NLTE cooling in the surface layers. The strengthening is rather modest but it can be larger if larger microturbulent velocity is assumed in the surface layers as may be suggested from Fig. 11. However, it is needed to find a velocity structure that explains consistently all the observed features such as shown in Figs. 11-13, and such a possibility should hopefully be explored. At present, however, our ad-hoc

lines by the contribution of the MOLsphere (for further details, see Tsuji 2008). 


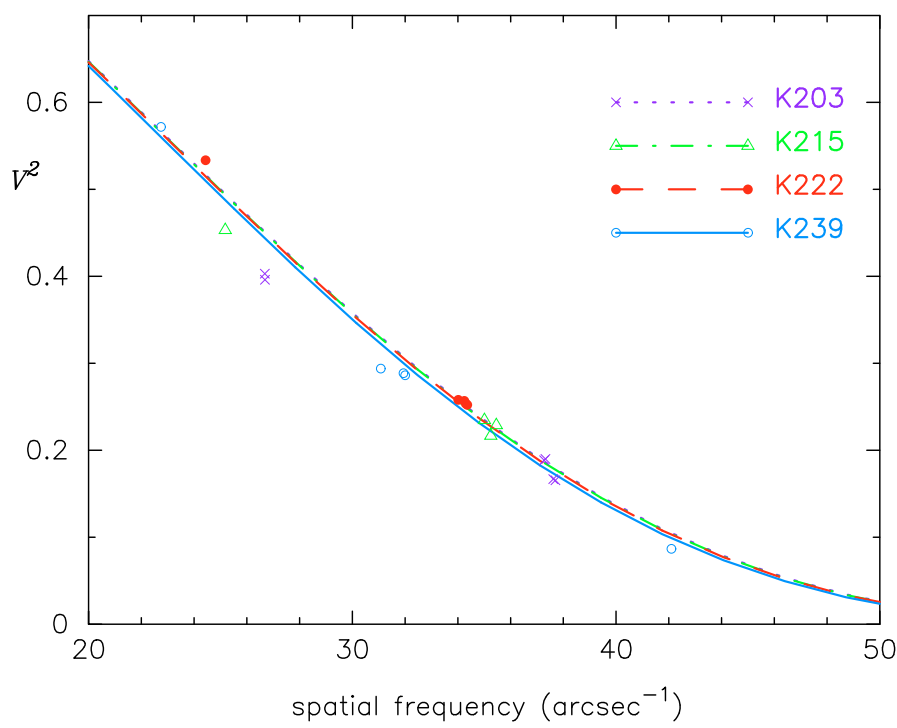

Fig. 15. Predicted visibilities squared based on the ad-hoc model discussed in the text are compared with the observed visibilities squared by Verhoelst (2005). The results for different filter bands are shown by different symbols and lines as noted in the figure. Predicted visibilities squared based on the RE model photosphere show only minor differences from those based on the ad-hoc model.

model is consistent with all these observed features as shown in Figs. 11-13. Also, relatively weak lines remain unchanged so far as photospheric models are applied and hence the weakening of the relatively weak lines cannot be explained by such models. By our ad-hoc model, however, the weakening can be explained as due to the thermal emission of the molecular clouds.

The pure rotation lines of $\mathrm{H}_{2} \mathrm{O}$ at $12 \mu \mathrm{m}$ were also observed in red supergiants such as $\alpha$ Ori (Ryde et al. 2006a) and $\mu$ Cep (Ryde et al. 2006b) in absorption, and it was difficult to understand why they were not detected in emission if water molecules reside in the extended outer envelope. Possibly for this reason, Ryde et al. (2006a,b) suggested that the water lines should originate in the photosphere. However, we know that the $\mathrm{H}_{2} \mathrm{O} v_{2}$ fundamentals at $6 \mu \mathrm{m}$ as well as the pure rotation lines at $40 \mu \mathrm{m}$ appear in emission in $\mu$ Cep (Tsuji 2000b). A possible way to resolve this dilemma can be our proposal that dust grains expected to condense in the envelope provide a continuum background (at around $12 \mu \mathrm{m}$ ) against which the $\mathrm{H}_{2} \mathrm{O}$ lines can be seen in absorption. However, we remained concerned that the dust grains may not able to be accommodated in the envelope, where radiation pressure can expel the dust grains (see note added in proof in Tsuji 2006). However, a detailed analysis of the infrared spectrum of $\alpha$ Ori possibly detected amorphous alumina in the extended envelope (Verhoelst et al. 2006). Also, clean dust grains such as alumina and silicate were shown not to suffer the effect of radiation pressure in full (Woitke 2006; Höfner \& Andersen 2007). Then, the $12 \mu \mathrm{m}$ water lines in absorption can also be produced by molecular clouds in the outer envelope of red supergiants. In other words, the $12 \mu \mathrm{m} \mathrm{H}_{2} \mathrm{O}$ absorption lines can consistently be understood as due to originating in molecular clouds in Arcturus as well as in red supergiants.

Finally, we examine whether our ad-hoc model can be constrained further by visibility measurements in four narrow-band filters, which show the wavelength dependence of the diameter of Arcturus (Verhoelst et al. 2005). Interferometric observations were completed in four narrow-band filters as for red supergiant star $\mu$ Cep (Perrin et al. 2005), and predictions of the visibilities based on models are carried out in exactly the same way as for $\mu$ Cep (Tsuji 2006). We use the stellar parameters in Table 3 and assume the angular diameter of the stellar photosphere to be 21.05 arcsec (Lacour et al. 2008) throughout.

The predicted visibilities squared based on our ad-hoc model are compared with the observed data in Fig. 15. First, we note that the results for the four bands show little difference, but the predicted visibility squared for $K 239$ band, which includes $\mathrm{CO}$ first overtone bands, indicates that the diameter for this band may be extended slightly. Second, some visibility data cannot be fitted well with the predicted visibilities, as noted already by Verhoelst et al. (2005). The poor fittings are not necessarily associated with the presence of molecular absorption in the filter bands and we conclude that this difficulty cannot be resolved by a simple model of the outer atmosphere.

The results for the RE photospheric model (SS model) are very similar to the results for the ad-hoc model. Since the extension of our ad-hoc model and hence its effect on visibilities are rather minor, the results in Fig. 15 may be evidence neither for nor against our ad-hoc model. The results of $\chi$-square test for the fits are: $\chi^{2} /(N-1)=11.60,12.40,1.71$, and 5.23 for $K 203, K 215, K 222$, and $K 238$ bands, respectively ( $N$ is the number of observed data points) for the ad-hoc model, and $\chi^{2} /(N-1)=10.93,11.82,2.13$, and 5.13 for $K 203, K 215$, $K 222$, and $K 238$ bands, respectively, for the RE photospheric model (SS).

\subsection{From Arcturus to cool luminous stars}

We noticed excess absorption in CO lines of M giant stars, first in the low excitation strong lines in late $\mathrm{M}$ giants. The excess absorption was difficult to interpret as being photospheric in origin, and we proposed instead that it originates from cool molecular layers (which are however warmer than the expanding circumstellar envelope) referred to as a quasi-static molecular dissociation zone (Tsuji 1988). We then found more or less similar unusual phenomenon not only in the low excitation strong lines but also in the intermediate-strength lines of $\mathrm{CO}$ and $\mathrm{OH}$ with LEP as high as $2 \mathrm{eV}$ in dozens of M-giant stars including K5 giant $\alpha$ Tau (Tsuji 2008). We have shown in this paper that this phenomenon can be recognized more simply by the curves-ofgrowth (Figs. 6-8). We have found in this paper that the same phenomenon appears in the early $\mathrm{K}$ giant $\alpha$ Boo and that the phenomenon can be seen more clearly in the lines of the $\mathrm{CO}$ fundamentals. Thus we have had to recognize that the unusual behavior of molecular lines is probably a more general phenomenon, which may be related to some basic property of cool stellar atmospheres.

In $\mathrm{M}$ giant stars, the intermediate-strength lines of unusual behavior showed little relative motion $\left(\$ 1 \mathrm{~km} \mathrm{~s}^{-1}\right)$ compared to the weak lines (Tsuji 1991), and they may also originate in molecular clouds formed within the outer atmospheres. We recall, however, that some strong low excitation lines $(\log W / v>$ -4.4 and LEP $\lesssim 0.5 \mathrm{eV}$ ) in the cooler $\mathrm{M}$ giants showed relative motions of a few $\mathrm{km} \mathrm{s}^{-1}$ (Tsuji 1988), which can be explained by some molecular clouds in the outer part (and hence cooler) beginning to decouple dynamically from the main bodies of the molecular clouds. We may refer to an aggregation of the molecular clouds, including those with low relative motions, as a MOLsphere for simplicity in $\mathrm{K}$ and $\mathrm{M}$ giants as well as in red supergiants throughout.

The spatial extension of the MOLsphere has already been demonstrated directly by observations of red supergiant stars with spatial interferometry (e.g. Perrin et al. 2005, 2007). The 
case of red supergiants shows a marked contrast to the case of Arcturus for which interferometry shows little evidence for an extended MOLsphere (Sect. 6.2). Clearly the details of the MOLsphere may differ significantly in different types of stars. The case of red supergiants may represent a more advanced phase in the evolution of molecular clouds discussed in the preceding paragraph for cooler $\mathrm{M}$ giant stars. The molecular clouds probably expand easily under the lower gravities of the outer atmosphere of supergiant stars.

In the case of Mira variables, gaseous matter can be easily levitated by pulsations and/or by shocks, and the nature of molecular clouds or MOLsphere may not necessarily be the same as in non-Mira variable stars. Anyhow the presence of the molecular shells in the outer atmospheres of Mira variables has clearly been shown by interferometric observations (e.g. Perrin et al. 2004b), by spectro-imaging (Le Bouquim et al. 2009), and by a differential spectral imaging with an adaptive optical system (Takami et al. 2009). The spectra of Mira variables have detected multiple velocity components, and the different layers related to different velocities have anomalous curves-of-growth (Sect. 4.4). This fact suggests that the molecular clouds may form in each layer of different velocities (and hence at different locations), and detailed analyses of spectral and interferometric observations of Mira variables will shed further light on the molecular clouds in the outer atmosphere of cool luminous stars.

\subsection{Formation of molecular clouds and origin of mass-loss}

We have considered a possibility that the molecular clouds or MOLsphere may exist through early $\mathrm{K}$ giants to coolest $\mathrm{M}$ giant stars. The major problem is how a large amount of gaseous material can be supplied to the outer atmosphere extended to a few stellar radii in the case of cooler (super)giant stars. In the case of Arcturus, the clouds may be formed not so far from the photosphere but the problem of how gaseous material can be transported there is the same. We have assumed a various kinds of surface activities (Sect. 6.1), but their detail is not specified. We now return to this subject for the case of non-Mira variable stars.

For this purpose, we recall that the surface activities are driven mainly by magnet-hydrodynamical (MHD) processes in stellar envelopes. For example, reappearance of dynamo activity may be due to convection in the sheared rotation layers generated between the spinning-up contracting core and spinningdown expanding envelope as the star evolves from the subgiant to the giant phase (Uchida \& Bappu 1982). This phenomenon, which occurs in the red giant phase, can explain the revival of the surface chromospheric activity due to the regenerated magnetic field, and lead to some material being injected into the outer atmosphere by associated surface activities.

A detailed MHD simulation of stellar winds in red giant stars starting from the photosphere in open magnetic field regions was performed by Suzuki (2007), who showed that the perturbations from the surface convection excite waves that propagate upward. An important result of Suzuki's simulation is that a nearly static region is formed at several stellar radii above the photosphere. The gaseous matter levitated by each MHD process will accumulate around the nearly static region and grow to become a reservoir of abundant gaseous material. This reservoir can be a seat of molecular cloud formation, in which the molecular cooling mechanism noted in Sect. 6.1 will also play a role. Such a reservoir has been detected observationally: We may identify the MOLsphere or the aggregation of molecular clouds formed in the outer atmospheres of early $\mathrm{K}$ to late $\mathrm{M}$ giants (Sect. 6.3) with the nearly static reservoir predicted by the MHD simulation.

Another important point is that the stellar winds are effectively accelerated from the nearly static region, or the reservoir, located at several stellar radii above the photosphere. Suzuki (2007) noted that this result explains why the observed flow velocity of the winds is rather low at about the escape velocity for several stellar radii above the photosphere. Thus, we conclude that the origin of stellar mass-loss in red giant stars has basically been resolved from theory and observations consistently. This result is particularly welcome in view of a lack of successful explanation for mass-loss mechanism in red giant stars, since previously proposed dust-driven mechanism was found not to be efficient enough to drive the mass-loss from oxygen-rich giant stars (Woitke 2006; Höfner \& Andersen 2007).

Generally, however, hydrodynamical processes including wave and shock formations result in rather violent variations in thermal and velocity structures of the outer atmosphere, which may have some observational consequences. On the other hand, violent phenomena are by no means evident in the observed data that we have examined, and further detailed consideration is needed to reconcile theories more successfully with observations. For example, each hydrodynamical event may have little observable effect if matter involved in a single event is rather small and only the accumulated effect may have observable effects.

\section{Concluding remarks}

We acknowledge the importance of observational data to our present work, in particular the excellent atlas of Arcturus (Hinkle et al. 1995). We were able to measure more than 100 lines of the $\mathrm{CO}$ first overtone bands based on the atlas spectrum in which telluric features had been effectively removed and, as a result, we found unexpected patterns in both our LL (Fig. 3) and CG (Fig. 5a) analyses of $\mathrm{CO}$ lines. On the other hand, we could measure only about 20 lines in a FTS spectrum from the KPNO archives, selecting only the lines undisturbed by the atmospheric lines and, as a result, we overlooked the anomalous behavior of the stronger CO lines (e.g. Fig. 1a in Tsuji 1986).

More importantly, many lines of the $\mathrm{CO}$ fundamentals have been made available by the atlas and we confirmed that the $\mathrm{CO}$ fundamental lines provide very excellent insight into the cool molecular constituent of Arcturus (see e.g. Figs. 4, 5b, 11, 12). The CO fundamental lines should certainly provide an excellent means of probing molecular clouds in cooler (super)giant stars, and analyses of the CO fundamental lines should hopefully be extended to many cool luminous stars, despite the well known difficulty caused by the strong atmospheric absorption in the $M$ band region where the $\mathrm{CO}$ fundamental lines are observed. High resolution spectroscopy of the $M$ band region from space would be ideal, although we are unaware of any such plans.

In our interpretation of the spectra, a major restriction is to have assumed LTE throughout. We have referred mainly to the detailed non-LTE analysis of CO line formation by Ayres \& Wiedemann (1989) and Wiedemann \& Ayres (1991). Departure from LTE should be certainly more important in the outer atmosphere and it is desirable that more or less similar NLTE analysis can be extended to the CO line formation in molecular clouds. Departure from LTE should also appear in other processes including atoms and other molecules. An example for some atoms (Short \& Hauschildt 2003) was already considered (Sect. 6.2), although the problem continues to exist because of the difficulty 
in UV fluxes having significant effects on excitation and ionization (Short \& Hauschildt 2009). It is a formidable task to take all the important processes consistently in a NLTE analysis and we hope further progress in this field.

Also, chemical processes in the molecular clouds should most probably not follow LTE. We estimated $\mathrm{CO}$ and $\mathrm{H}_{2} \mathrm{O}$ column densities in the molecular clouds and $\mathrm{H}_{2} \mathrm{O} / \mathrm{CO}$ ratio appeared to be $4.0 \times 10^{-3}$ (Sect. 6.2). At $T=2000 \mathrm{~K}$, this ratio corresponds to an equilibrium value at $\log P_{\mathrm{g}}=-1.63$, which may not be very unreasonable for $P_{\mathrm{g}}$ in the outer atmosphere (note that $\log P_{\mathrm{g}} \approx-0.5$ at the surface of our photospheric model shown in Fig. 2). However, this result of course does not prove the validity of LTE assumption in molecular clouds. Without a better method, however, we have assumed LTE to estimate abundances of some other molecules of interest, for example TiO. For $T=2000 \mathrm{~K}$ and $\log P_{\mathrm{g}}=-1.63, \mathrm{TiO} / \mathrm{CO}$ is $4.09 \times 10^{-4}$. Then TiO column density is about $2 \times 10^{+16} \mathrm{~cm}^{-2}$, which is not high enough to provide TiO features as strong as those noted by Ryde et al. (2003) for their cool photospheric model. For predicting molecular abundances in molecular clouds, detailed analysis of non-equilibrium processes are required, and we hope increasingly sophisticated approaches will be applied as more detail about the molecular clouds can be clarified.

In this paper, we concluded that the infrared spectra of cool luminous stars cannot be understood as originating in the photosphere alone, but should have hybrid nature due to at least two components: a photosphere and a slightly cooler but not very cool molecular clouds or MOLsphere. We noticed that ignorance of the hybrid nature of the infrared spectra results in a large systematic error in abundance determinations based on molecular lines (Tsuji 2008). Other authors (e.g. Decin et al. 2003) also noticed that the observed infrared spectra of red giant stars, including Arcturus, are not necessarily matched very well by the predicted ones based on the most recent model photospheres. We suggest that such a difficulty may also be related to the hybrid nature of the infrared spectra, at least partly.

By the way, we have found that the classical curve-of-growth method provides a simple means by which to recognize the hybrid nature of the infrared spectra. The CG method can easily be applied to any object only if EWs of some dozens of lines can be measured. What is important is to measure as many lines as possible, including the weak and the intermediate-strength lines. We hope that this simple CG method will be applied to other objects (e.g. carbon stars) and/or to other spectral lines (e.g. TiO, atomic lines) to clarify the nature of the hybrid spectra in more detail. Also, the simple CG method is effective when spectral lines consist of the contributions from different components as in the CO fundamental lines (Sect. 4.1).

Given that the molecular clouds have low global motions and rather similar temperatures relative to the surrounding atmosphere, they could be recognized only by subtle spectroscopic signatures. For this very reason, the possible presence of such a molecular constituent has been overlooked for a long time. This is in marked contrast to the case of a hot chromosphere, which could be recognized in terms of the strong emission lines, and an expanding circumstellar envelope recognized by the blue-shifted zero-volt lines at an early time (Adams \& MacCormac 1935). Now do clouds exist in the atmosphere of Arcturus? It is certainly not easy to answer this question by spectroscopic observations alone. But we found several arguments in favor of the presence of molecular clouds in Arcturus in this paper. Also, in cooler luminous stars in which $\mathrm{CO}$ lines show unusual behaviors as in Arcturus (Sect. 6.3), the presence of molecular clouds has been shown more directly by observations with spatial interferometry (e.g. Mennesson et al. 2002; Perrin et al. 2004a). We hope further progress in sensitivity as well as in resolution in future stellar interferometry so that clouds in stellar atmospheres can be resolved.

The outer layers of red giant stars are quite complicated consisting of many different components, and the definition of each component is by no means clear. For example, the terms "atmosphere" and "photosphere" are often confused. Probably, "atmosphere" may involve all the observable outer layers of a star while "photosphere" represents the base of the atmosphere where continuum radiation and most absorption lines are formed. We have no model of an "atmosphere" for red giant star yet, even if we have some models of the "photosphere". In the literature, such a "model photosphere" is often referred to as "model atmosphere" but, strictly speaking, this does not represent the real situation.

Thus, we have no model atmosphere of cool luminous stars yet and, moreover, the picture of stellar atmospheres might have been too simplified until the present. For example, it may be an over-simplification to assume that the stellar atmospheres are completely free of clouds. Cloud formation is a rather common feature in celestial objects that we know in detail (e.g. our Earth and planets). The dust clouds are also common in brown dwarfs and dust cloud formation in ultracool dwarfs is now under intensive investigations by different approaches (e.g. Helling et al. 2008). Molecular cloud formation in cool luminous stars is an important topic that should be pursued in more detail, since it can be one of the basic processes in cool stellar atmospheres, and should have important effects on many problems from interpretation of the spectra to the origin of mass-loss. Molecular clouds in cool luminous stars may encompass masering clouds observed in OH-IR stars, and thus cloud formation should play a key role in understanding various phenomena observed in all the family of cool luminous stars.

A new problem that we have encountered is how the molecular clouds, referred to as MOLsphere for convenience in modeling, can be formed in the outer atmosphere of cool luminous stars. The MOLsphere differs from the so-called CO-mosphere if the CO-mosphere simply represents the cooler component of the bifurcated thermal structure, while the MOLsphere should consist of molecular clouds formed from additional gaseous material (Sect. 6.1). Thus, the first problem is to explain how additional material can be supplied to the outer atmosphere. To push additional material into the upper atmosphere, magnetic forces due to MHD processes appeared to be promising (Sect. 6.4), and we hope that there will be further progress in this field. The second problem to be solved is how clouds form with the material supplied by dynamical processes. In an environment where molecules and dust form easily (i.e. at rather low temperatures in cool stellar atmospheres), cloud formation is possible, for example, by cooling instability induced by the molecular formation itself (Sect. 6.1). Thus it seems to be more natural to assume that clouds form in the atmosphere of cool stars than to assume that the atmosphere remains free of any cloud. However, further detail of these processes should be worked out.

To answer the question "Is Arcturus a well-understood K giant?" (Verhoelst et al. 2005), we unfortunately cannot answer positively at present. This question was addressed in connection with the near-IR interferometric observations, but spectroscopic observations are by no means better understood yet. For observations such as the unexpected detection of $\mathrm{H}_{2} \mathrm{O}$ pure rotation lines (Ryde et al. 2002) or unpredictable upturn of the flat part of the curves-of-growth for CO lines (Fig. 5), we have no definite guidelines by which to interpret the observed data. The situation 
is more or less the same for other cool luminous stars, and relatively well-observed Arcturus will serve as invaluable reference for the interpretation and analysis of other cool luminous stars for which observations are more limited. Thus, we have sufficient reasons why we study Arcturus carefully.

Acknowledgements. I thank Takeru K. Suzuki for helpful discussion on stellar mass-loss and related problems in red giant stars. I am much indebted to an anonymous referee for invaluable suggestions and many helpful comments in improving the text. My thanks are also due to Kenneth H. Hinkle for making available the electronic version of the Arcturus Atlas at an early time. Data analyses were in part carried out on common use data analysis computer system at the Astronomy Data Center (ADC) of NAOJ and this research has made use of the VizieR catalogue access tool, CDS, Strasburg, France. This work was supported by Grant-in-Aid of JSPS for Scientific Research (C) nos.17540213 \& 21540237.

\section{References}

Adams, W. S., \& MacCormac, E. 1935, ApJ, 81, 119 Ayres, T. R., \& Linsky, J. L. 1975, ApJ, 200, 660

Ayres, T. R., \& Wiedemann, G. 1989, ApJ, 338, 1033

Chackerian, C. Jr., \& Tipping, R. H. 1983, J. Mol. Spectrosc., 99, 431

Cuntz, M., \& Muchmore, D. O. 1994, ApJ, 433, 303

Decin, L., Vandenbussche, B., Waelkens, C., et al. 2003, A\&A, 400, 679

Dravins, D. 1987, A\&A, 172, 221

ESA 1997, The Hipparcos and Tycho Catalogues, ESA SP-1200, Noordwijk

Griffin, R. F. 1968, A photometric atlas of the spectrum of Arcturus, $\lambda \lambda$ 36008825 A (Cambridge: Cambridge Phil. Soc.)

Griffin, R., \& Griffin, R. 1973, MNRAS, 162, 243

Griffin, R. E. M., \& Lynas-Gray, A. E. 1999, AJ, 117, 2998

Guelachivili, G., De Villeneuve, D., Farrenq, R., Urban, W., \& Verges, J. 1983, J. Mol. Spectros., 98, 64

Heasley, J. N., Ridgway, S. T., Carbon, D. F., Milkey, R. W., \& Hall, D. N. B. 1978, ApJ, 219, 970

Helling, Ch., Ackerman, A., Allard, F., et al. 2008, MNRAS, 391, 1854

Hinkle, K. H. 1978, ApJ, 220, 210

Hinkle, K. H., \& Barnes, T. G. 1979, ApJ, 227, 923

Hinkle, K. H., Lambert, D. L., \& Snell, R. L. 1976, ApJ, 210, 684

Hinkle, K. H., Hall, D. N. B., \& Ridgway, S. T. 1982, ApJ, 252, 697

Hinkle, K., Wallace, L., \& Livingston, W. 1995, Infrared Atlas of the Arcturus Spectrum, 0.9-5.3 microns (San Francisco: Astron. Soc. Pacific)

Höfner, S., \& Andersen, A. C. 2007, A\&A, 465, L39
Jacquinet-Husson, N., Arié, E., Ballard, J., et al. 1999,

J. Quant. Spec. Radiat. Transf., 62, 205

Lacour, S., Meimon, S., Thiébaut, E., et al. 2008, A\&A, 485, 561

Lambert, D. L., Brown, J. A., Hinkle, K. H., et al. 1984, ApJ, 284, 223

Le Bouquin, J.-B., Lacour, S., Renard, S., et al. 2009, A\&A, 496, L1

Mennesson, B., Perrin, G., Chagnon, G., et al. 2002, ApJ, 579, 446

Muchmore, D. O., Nuth, J. A., \& Stencel, R. E. 1987, ApJ, 315, L141

Partridge, H., \& Schwenke, D. W. 1997, J. Chem. Phys., 106, 4618

Perrin, G., Ridgway, S. T., Coudé du Foresto, V., et al. 2004a, A\&A, 418, 675

Perrin, G., Ridgway, S. T., Mennesson, B., et al. 2004b, A\&A, 426, 279

Perrin, G., Ridgway, S. T., Verhoelst, T., et al. 2005, A\&A, 436, 317

Perrin, G., Verhoelst, T., Ridgway, S. T., et al. 2007, A\&A, 474, 599

Peterson, R., Dalle Ore, C. M., \& Kurucz, R. L. 1993, ApJ, 404, 333

Quirrenbach, A., Mozrukewich, D., Armstrong, J. T., Buscher, D. F., \& Hummel,

C. A. 1993, ApJ, 406, 215

Ryde, N., Lambert, D. L., Richter, M. J., et al. 2002, ApJ, 580, 447

Ryde, N., Lambert, D. L., Richter, M. J., Lacy, J. H., \& Greathouse, T. K. 2003, in 3 D Stellar Evolution, ed. S. Turcotte, S. C. Keller, \& R. M. Cavallo (San Francisco: Astron. Soc. Pacific), ASP Conf. Ser., 293, 214

Ryde, N., Harper, G. M., Richter, M. J., Greathouse, T. K., \& Lacy, J. H. 2006a, ApJ, 637, 1040

Ryde, N., Richter, M. J., Harper, G. M., Eriksson, K., \& Lambert, D. L. 2006b, ApJ, 645, 652

Short, C. I., \& Hauschildt, P. H. 2003, ApJ, 596, 501

Short, C. I., \& Hauschildt, P. H. 2009, ApJ, 691, 1634

Suzuki, T. K. 2007, ApJ, 659, 1592

Takami, H., Goto, M., Gaessler, W., et al. 2009, PASJ, in press [arXiv: 0904.2570v1]

Tsuji, T. 1978, A\&A, 62, 29

Tsuji, T. 1986, A\&A, 156, 8

Tsuji, T. 1988, A\&A, 197, 185

Tsuji, T. 1991, A\&A, 245, 203

Tsuji, T. 2000a, ApJ, 538, 801

Tsuji, T. 2000b, ApJ, 540, L99

Tsuji, T. 2001, A\&A, 376, L1

Tsuji, T. 2006, ApJ, 645, 1448

Tsuji, T. 2008, A\&A, 489, 1271

Uchida, Y., \& Bappu, M. K. V. 1982, JA\&A, 3, 277

Unsöld, A. 1955, Physik der Sternatmosphären mit Besonderer Berücksichigung der Sonne, 2ten Auf. (Berlin: Springer)

Verhoelst, T., Bordé, P. J., \& Perrin, G., et al. 2005, A\&A, 435, 289

Verhoelst, T., Decin, L., Van Malderen, R., et al. 2006, A\&A, 447, 311

Wiedemann, G., \& Ayres, T. R. 1991, ApJ, 366, 277

Wiedemann, G., Ayres, T. R., Jennings, D. E., \& Saar, S. H. 1994, ApJ, 423, 806

Woitke, P. 2006, A\&A, 460, L9

Woolf, N. J., Schwarzschild, M., \& Rose, W. K. 1964, ApJ, 140, 833 